\title{
ESTORIA DEL FECHO DE LOS GODOS
}

\author{
Manuel HiJANo VILLEGAS \\ University of Durham
}

\section{INTRODUCCIÓN}

Una de las últimas metamorfosis sufridas en el transcurso de los siglos bajomedievales por la Estoria de España (EE) mandada componer por Alfonso $X$ es la que representan las distintas versiones de la Estoria del fecho de los godos (Efg), crónica compilada en el siglo XV y muy difundida, a juzgar por el número de manuscritos conservados y por su empleo como fuente por varios historiadores de finales del XV y del XVI. En este trabajo me propongo complementar el conocimiento que sobre este modelo cronístico tenemos gracias, sobre todo, a la labor de Diego Catalán, autor en 1966 del estudio definitivo sobre esta obra ${ }^{3}$. También quisiera ofrecer una serie de reflexiones respecto al valor que este tipo de compilaciones tardías pueden tener, no ya como testimonio del texto original alfonsí, sino como fuente de datos sobre las prácticas y métodos de trabajo de los compiladores y copistas de crónicas y, cn definitiva, sobre la difusión del discurso histórico durante el final de la edad media y el principio de la moderna.

"Estorias del fecho de los godos" es la denominación que Diego Catalán asigna a dos modelos cronísticos caracterizados por combinar el texto de * $\mathbf{L}$ ', ejemplar perdido de una "Crónica general" representada también por el ms. L $(=1298$, Bibl. Nacional), con la sección correspondientc a la "Historia gótica" del Toledano romanzado $(T R)$, una traducción, probablemente tardia, del De rebus Hispaniae $(D R H)$ del arzobispo de Toledo Rodrigo Jiménez de

\footnotetext{
' Cito por el trabajo de 1966 c incluyo además la referencia en Catalán 1992: 121-37 y 231-85, donde su autor lo refunde y actualiza. Véanse también las descripciones de la crónica en los trabajos de Fernández Ordóñez (2000: 257-58), Crespo (2000: 122) y, sobre todo, María del Mar Bustos (2002), aunque la investigadora desconoce algunos de los testimonios de la crónica presentados en estc trabajo.
}

Revista de Literatura Medieval, xx (2008), pp. 2 II-24I.

ISSN: 1130-3611 
$\mathrm{Rada}^{2}$. Ambos modelos, llamados por Catalán Estoria amplia $(E A)$ y Estoria breve $(E B)$, atendiendo a la mayor cxtensión que el primero de ellos otorga a la fuente alfonsí, coinciden también en completar el relato del $T R$, a partir del punto en que el arzobispo lo dejó tras la conquista de Córdoba por Fernando III (1242), con un mismo "Seguimiento del Toledano" que da cuenta de los reinados siguientes mediante la adición y enlace de varias fuentes de origen diverso: la llamada Historia hasta 1288 dialogada para el final del reinado de Fernando III y los reinados de Alfonso $x$ y Sancho IV hasta 1288; un atropellado relato del final del reinado de Sancho IV y el reinado de Fernando IV, que parece un arreglo del compilador para enlazar con la materia siguiente; un resumen (¿también obra del compilador?) de la Crónica de Alfonso $X I$, completado con una prosificación de los últimos versos del Poema de Alfonso Onceno y, por último, unos Anales antiguos sevillanos que abarcan los reinados a partir de Pedro I hasta, al menos, el primer año de Juan II (1407). Catalán (1966: 51-64 [=1992: 121-37]) tambićn se ocupó de caracterizar la crónica testimoniada por * $\mathbf{L}$ ' y $\mathbf{L}$ como un texto mixto, fruto de yuxtaponer los textos de las versiones Vulgar hasta Eurico (VV), Enmendada después de 1274 (VE) y Crítica (VC) dc la Estoria de España.

\section{TESTIMONIOS}

A continuación ofrezco una relación de todos los testimonios conservados de la Estoria del fecho de los godos para luego detenerme a comentar con más detenimiento los manuscritos que Diego Catalán no consultó o, en un caso concreto, atribuyó un carácter erróneo. Incluyo las referencias a los testimonios en Menćndez Pidal (1898), Sánchez Alonso (1925), Catalán (1966, 1992, 1997 y 2005), Fernández Ordóñez (1993), Bustos (2002) y Faulhaber et al (1984). Empleo las siglas Bi, C y Li para referirme a estos manuscritos con más comodidad.

\footnotetext{
${ }^{2}$ El Toledano romanzado fue sacado a la luz por Gómez Pćrez (1962), quien erróncamente identificó el texto con la traducción del $D R H$ cmpleada por los historiadores alfonsies. Catalán analizó el texto y seîaló su auténtico carácter en los dos trabajos ya citados (1966: 11-31; 1992: 61-91) y, de nuevo, en un libro reciente (2005: 633-58). He consultado el texto del TR a travćs de la edición realizada por Carol Anne Van der Wait con motivo de su tesis doctoral, la cual reproduce la sección de la "Historia gótica" del ms. H (Real Academia de la Historia, 9-30-7 / 6511 [olim 11.12: fa 2\%/63; 10.8.4]), uno de los dos testimonios "puros" del romanceamiento. En cuanto al texto latino de Jiméncz de Rada, lo consulto por la edición de Fernández Valverde (1987).
} 
B Biblioteca Nacional de Madrid, 9563 (olim Bb 87). 135 folios. Siglo XV. Comienza trunco en la historia de los jueces de Castilla. Completo por el final, incluyendo las noticias de los años 14101430 ("Adición de Moguer"). Cfr. Sánchez Alonso 1925: 349; Catalán 1966: 33 y nn. 86-8 [=1992: 233 y nn. 7-9]; 1997: 483 (como Efg-B); Fernández Ordóñez 1993: 327 (como L-B); Bustos 2002: 482; Faulhaber et al 1984: ref. 1624.

Bi Birmingham University Library, 5-IV-22 (olim Oxford, Dolphin Book Co., Cat. 50, n. 13). 278 folios. Finales del Xv. Falto de su primer folio y de sus folios finales. Cfr. Faulhaber et al 1984: ref. 2447.

C Biblioteca Universitaria de Sevilla, 331-143. 203 folios. Siglo XV. Íntegro por el principio y por el final. Termina con la "Adición de Moguer”. Cfr. Faulhaber et al 1984: ref. 3077; Catalán 2005: 646, n. 26.

D Biblioteca Nacional de Madrid, 9559 (olim Dd 179). 229 folios de los que el texto de la crónica ocupa los primeros 227. Siglo XV. Representante de una Estoria amplia del fecho de los godos refundida después de 1455. Cfr. Catalán 1966: 35 y nn. 95-8 [=1992: 234 y nn. 16-19, y 334]; 1997: 483 (como Efg-D); Fernández Ordóñez 1993: 268-69 y 328 (como L-D); Bustos 2002: 483; Faulhaber et al 1984, ref. 1622.

D- Edición del Marqués de la Fuensanta del Valle publicada en 1893 ed en los volúmenes 105 y 106 de la Colección de Documentos Inéditos para la Historia de España con el título Crónica de España del arzobispo Don Rodrigo Jiménez de Rada. Tradújola en castellano y la continuó hasta su tiempo Don Gonzalo de la Hinojosa y después un anónimo hasta el año de 1454. Bibli Nac.-Dd-179. Reproduce (con varios errores) el texto del manuscrito $D$. El volumen 105 (D-ed $)_{1}$ contiene el relato hasta el final de la historia de Jiménez de Rada, el 106 (D-ed $)_{2}$ la continuación (3-141), que erróneamente se atribuye al obispo de Burgos Gonzalo de la Hinojosa por ser el autor mencionado en la portada y en una nota marginal del manuscrito (posteriores ambas a la copia). Cfr. Menéndez Pidal 1898: 93-4; Catalán 1966: 37, n. 98 y 36, n. 102 [=1992: 234 y n. 19]; 1997: 483 (como Efg-D-ed); Fernández Ordóñez 1993: 269; Bustos 2002: 483-84 ${ }^{3}$.

\footnotetext{
${ }^{3}$ En las citas del texto de la $E A$ incluyo la referencia a la cdición de 1893 , empleando las siglas D-ed, para el volumen 105 y D-ed 2 para el 106, seguidas del número de página y línea, aunque preficro citar el texto del ms. Bi, base de mi propia edición (2003). El texto de Fucnsanta del Valle presenta dos discrepancias importantes con respecto al del ms. D. En primer lugar, no reproduce el final del capitulo correspondiente a la traducción de la "divisio orbis tripartita" del $T R$ (D-ed 1 : cap. V, 13 15 cfr. D, ff. 4v-Sr), a partir de "en Africa son doze prouinçias..." y funde su texto con cl del capitulo siguientc, cuyo epigrafe ("Dcl quinto fijo de Jafet que fue Tubal \& commo sus fijos poblaron a España") omite. El capitulo siguiente va numerado, sin embargo, como sćptimo, de acuerdo con D (D-ed 1 : $15_{25}$ ). El editor tambićn omite sin razón aparente el texto del capitulo correspondiente al año $9^{\circ}$ de Alfonso I (D, ff. 69v-70r) del que sólo reproduce el epígrafe ("De lo que avino en el noveno año del rey don
} 
F Biblioteca Nacional de Madrid, 1517 (olim F 133). 326 folios. Siglo XVI. Cfr. Catalán 1966: 34 y n. 89 [=1992: 233-34 y nn. 1012]; 1997: 483 (como Efg-F); Fernández Ordóñez 1993: 327 (como L-F); Bustos 2002: 482.

F' Biblioteca Nacional de Madrid, 1295 (olim F-60 bis). 377 folios. Siglo Xvil. Copia de F. Lleva el título "Coronica de España del infante don Juan Manuel". Cfr. Sánchez Alonso 1925: 348; Catalán 1966: 34 y n. 91 [=1992: 234 y n. 12]; Bustos 2002: 482.

I Biblioteca Nacional de Madrid, 10154, (olim Ii 60). 388 folios. Siglo XVI. Copia de $\mathbf{L}$ pues conluye en el final trunco de dicho manuscrito. Fechado en 1571. Cfr. Sánchez Alonso 1925: 349; Catalán 1966: 34 y notas. 93-4 [ =1992: 234 y n. 14]; 1997: 483 (como Efg-I); Fernández Ordóñez 1993: 328 (como L-I); Bustos 2002: 482-83.

LI Biblioteca de la Real Biblioteca de Madrid, 2-LL-2. Siglo XVI. Falto de sus folios finales. Cfr. Menéndez Pidal 1898: 97-98, ms. $\mathrm{n}^{\circ}$ 27 (como D'); Catalán 1966: 34 y nn. 92-94; 1992: 334; 1997: 483 (como Efg-Ll); Femández Ordóñez 1993: 327 (como L-L); Bustos 2002: 483.

Estoria breve del fecho de los godos

A Biblioteca de Castilla-La Mancha en Toledo, 131. 402 folios. Copia fechada en 1545 realizada para la Duquesa de Alba. Texto completo por el comienzo y por el final. Finaliza con la misma “Adición de Moguer" que B y C. Cfr. Catalán 1966: 33, n. 88; 1992: 233, n. 9.

M' Biblioteca Nacional de Madrid, 3060, (olim M 289). Siglo XVIII. Copia o descriptus de $\mathbf{T}$, pues su texto concluye en el final trunco de dicho manuscrito e incluye su Coronica de los thurcos. Cfr. Catalán 1966: 33, n. 85 (como M) [=1992: 233 y n. 6]; Bustos 2002: 482.

Li Biblioteca Ajuda de Lisboa, 49-XI-37. 235 folios. Siglo XVII. Presenta el mismo comportamiento al final que $\mathbf{M}^{\prime}$, por lo que también debe derivar de T. Cfr. Faulhaber et al 1984: ref. 765.

S Biblioteca Nacional de Madrid, 6429 (olim S 55). 283 folios. Siglo XVI. Acéfalo por pérdida de sus primeros 10 folios. Cfr. Sánchez Alonso 1925: 346; Catalán 1966: 32 y n. 83 [ =1992: 232 y n. 4]; 1997: 483 (como Efg-S); Fernández Ordóñez 1993: 327 (como L-S); Bustos 2002: 481-82; Faulhaber et al 1984: ref. 1481.

T Biblioteca Nacional de Madrid, 7074 (olim T 29). 141 folios. Siglo XV. La crónica acaba trunca en el folio $114 \mathrm{v}$ hacia el final del

\footnotetext{
Alonso el Católico") y las refercncias cronológicas hasta "e el imperio de Constantyn en", tras lo que ofrece "diez" en vez de "çinco años", que es la lección del manuscrito y, a continuación, saita al texto del capítulo siguiente, correspondiente al año decimocuarto de Alfonso I, desde "Isca miramomelin cn somo de su tiempo...". A partir de ahi la numeración de capítulos en la edición irá una cifra por detrás de la del manuscrito, que es ya de por sí errática (D-ed, $: 238_{27}-239_{1}$ cfr. D, ff. 69v-70r).
} 
primer capítulo dedicado a Fruela II. Los folios 115r-141r contienen una Coronica de los thurcos, escrita por mano distinta y probablemente añadida por el formador del códice. Cfr. Sánchez Alonso 1925: 346; Catalán 1966: 33 y n. 84 [=1992: 233 y nn. 56]; 1997: 484 (como Efg-T); Fernández Ordóñez 1993: 327 (como L-T); Bustos 2002: 482.

En total se conservan trece testimonios manuscritos de las Estorias, cuatro de los cuales son descripti o copia dirccta de manuscritos existentes ( $F^{\prime}$ copia de F, I de $\mathbf{L l}$ y $M^{\prime}$ y $L i$ de $\left.T\right)^{4}$. A éstos hay que añadir tres manuscritos representantes de compilaciones que emplcaron el texto de la $E f g$ (o el del TR) para completar lagunas en sus respectivos prototipos de Crónica general ${ }^{5}$. La mayoría de los manuscritos presentan finales defectuosos, bien por pérdida de sus folios finales, en los casos de Bi, Ll, S y T, bien por copiar ejemplares incompletos, en los casos de F, I (copia de LI), M' y Li (los dos últimos copias de T). Los manuscritos completos por el final son B, C y D de la $E A$ y $A$ de la $E B$. D es representante de una Refundición de la $E A$ rcalizada poco después de 1455 caracterizada por reemplazar los capítulos originales dedicados a Pedro I y Juan II por un relato de dichos reinados similar al de la Refundición del Sumario del Despensero de la reina doña Leonor ${ }^{6}$. Por otro lado, las noticias relativas al periodo $1410-30$ con que conluyen $A$, B y $\mathbf{C}$ pudieran ser una actualización al relato original, lo cual

\footnotetext{
${ }^{4}$ Otro testimonio es el ms. 10614 (olim Kk-32) de la Biblioteca Nacional de Madrid. Se trata de una copia firmada por Miguel de Salvá cl 20-3-1840 de un códice del siglo XVI titulado "Varias historias", que según su autor se encontraba en la biblioteca de la Real Academia Española. El texto es similar a F y F', aunque acaba al final del reinado de Enrique II. Cfr. Sánchez Alonso 1925: 349; Catalán 1966: 34, n. 91 [=1992: 234, n. 12]; Bustos 2002: 483.

${ }^{5}$ Se trata de los sigujentes mss.: M.- Biblioteca Nacional de Madrid, 642 (olim G-170). Siglo XV. Cfr. Catalán 1966: 70-71 [=1992: 294-95]. Se inicia, trunco de sus folios iniciales, con cl texto de la Efg (o cl del TR) a partir del matrimonio de Fernando III con dona Juana de Pontiz pero llegado al final del relato del arzobispo enlaza con el "Seguimiento" propio de la Crónica particular de San Fernando. El formador del texto probablementè tuviera en mente el modelo de dicha crónica particular cuando realizó su labor. Según informa Catalán (1966: 70, n. 229 [=1992: 294, n. 43]), cl manuscrito es cl original del ms. 13002, también en la Biblioteca Nacional. O-F.- Biblioteca Nacional de Madrid, 828 (olim F-21). Siglo XVI. Formado por varias manos, en su texto sc mezclan los de la Versión regia, la Crónica vulgata y la Efg. Cfr. Catalán 1966: 72-73 [1992: 296-97] (como F) y 1997: 488. O-R.- Biblioteca de Palacio, 11-2038 (olim 2-N-4). Testimonio del sigło XV de la Crónica general vulgata completado por un adicionador del siglo XVI con un fragmento de la $E f g$, desde la mucrte de Sancho el Mayor de Navarra hasta cl capitulo posterior al de la muerte de Alfonso VI, donde acaba trunco. Cfr. Menéndez Pidal 1898: 98, ref. 28; Catalán 1966: 71-72 [=1992: 295-96] (como R); 1997: 488 (como O-R). Sobre estos manuscritos, vid. también Bustos 2002: 480 y 485 .

${ }^{6}$ Cfr. Catalán 1966: 89-99 [=1992: 262-83]. Vid. el texto de los dos nuevos capitulos en D-ed2: 69-98 y 109-41, y compárese con el de la Refundición que se puede leer en Rodriguez de Cuenca 1781. Catalán (1966: 89-99 [ $=1992: 272-83]$ concluye que D y la Refundición del sumario reproducen independientemente una crónica perdida, posible obra de Alonso Díaz de Montalvo, jurista durante los reinados de Juan II, Enrique IV y los Rcyes Católicos.
} 
plantea los problemas para la datación de la crónica que comentaré en la sección 6.

Otro testimonio de la $E f g$, al que nos referiremos después, es el que aporta un breve Sumario, probablemente de la $E A$, contenido en el ms. 7404 de la Biblioteca Nacional (olim T 211. Siglo XVII. 26 folios, de los que el texto ocupa los 12 finales. Cf. Sánchez Alonso 1925: 352). También contamos con testimonios indirectos en dos obras historiográficas de finales del $\mathrm{XV}$ que emplearon la $E f g$ como fuente: cl Repertorio de príncipes del alcalde de Jaén Pedro de Escavías (Garcia, 1972) y la Crónica abreviada del Mosén Diego de Valera ${ }^{7}$. Por último, la sección relativa a la historia de los Infantes de Salas alcanzó gran difusión como parte de una Crónica del noble cauallero el conde Fernán González, obrita impresa en repetidas ocasiones a lo largo del siglo XVI (Bustos 2002: 480-81).

3. TESTIMONIOS "NUEVOS" DE LAS ESTORIAS DEL FECHO DE LOS GODOS

La principal novedad que esta lista aporta respecto a la ofrecida por Diego Catalán en 1966 y 1992 es la aparición de dos "nuevos" manuscritos de la $E A$ (Bi y C) y el establecimiento del verdadero carácter del ms. A, como representante, el más completo del que disponemos, de la $E B$. Aunque estos manuscritos no alteran radicalmente los stemmas esbozados por otros investigadores (Fernández Ordóñez 2000: 258), si amplían nuestro conocimiento sobre la filiación entre los manuscritos y ofrecen algún dato nuevo en cuanto a la composición y el carácter de los respectivos modelos compilatorios de las Estorias.

Bi es un claro ejemplar de la $E A$. Aunque falto de la materia relativa a Juan II por pérdida de sus folios finales, contiene cl capítulo dedicado a Pedro I propio de los Anales sevillanos con que con-

'Es evidente que Escavias cmplé un ejemplar de la $E A$ con la "Adición de Moguer" para la historia a partir del final del reinado de Fernando III hasta cl año $1410 \mathrm{en}$ el reinado de Juan Il (Garcia 1972: 257-325). Pero ya se observa la consulta de la EA en ciertos detalles de la historia anterior, como la referencia a Alarigo como "fijo del rey Eurico e de la reyna Amalasent" (64), o la noticia, propia de la VE, L y la EA, del traslado de los restos de Bamba de Pamplicga a Toledo por Alfonso X (99-100). De la misma manera, la Crónica abreviada (CA) coincide en muchos de sus detalles con la EA. Por cjemplo, Valera da la noticia del traslado del cuerpo de Bambe (CA, III, xxii); llama "Egica" y no "Oppa" al arzobispo traidor que habló con Pelayo en la cueva $(C A, I V, i)$, como hace la $E A$ frente a $L$ y la $V C$, y no narra narra el tragico final de le epopeya de Bemardo del Carpio típico de las crónicas gencrales, simo el "final feliz" propio del arzobispo que ofrecen la $E f g$ y el $T R$ (CA, IV, xii). Como ocurre en el caso de tiscavias, el relato desde Fernando III hasta el año $1410(\mathrm{CA}$, IV, exii-cxxiv) pone en evidencia que Valera consultó un cjemplar de la Efg que acababa con la "Adición de Moguer" (al menos hasta la noticia de la toma de Antequera) ipodría ello indicar una relación entre la crónica de Escavias y la de Valera? El capitulo de la Valeriana dedicado a Juan Il puede leerse en Mata Carriazo 1941: $301-05$. 
cluye la $E A$ y no el característico de la $E A$ refundida después de 1455 representada por D. Al mismo tiempo, Bi se agrupa con $\mathrm{F}$ y $L I$ frente a D y C, manuscritos que, como en seguida veremos, interpolan un texto de fuente desconocida en el capítulo sobre las amazonas y en los siguientes reemplazan el texto de ${ }^{\prime} \mathbf{L}$ ' por el del

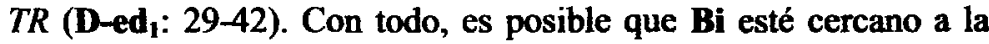
rama de la que derivan $C$ y $D$, ya que en esa misma sección presenta una laguna, aunque el texto perdido es menos extenso que el sustituido en $\mathbf{C}$ y $\mathbf{D}^{8}$. Por otro lado, Bi desconoce el error que en los primeros capítulos de la historia goda presentan $\mathbf{F}$ y Ll, manuscritos descendientes de un ejemplar de la $E A$ que tenía un folio encuadernado al revés (con el verso en el lugar del recto). Bi también ofrece lagunas y errores que le son propios?.

Mayor importancia para el estudio de la tradición textual de la Efg tiene $\mathbf{C}$, único manuscrito completo, por el principio y por el final, de la $E A^{10}$. C cierra su relato con la "Adición de Moguer has-

\footnotetext{
"La laguna de Bi abarca desde la mitad de su capitulo I, xvi (Tolefo, Euripilo y Tomaris cfr. PCG, cap. 391) hasta las primeras líncas del capitulo I, xx (los godos en tiempo de Damaciano, cfr. $P C G$, cap. 395). La "mano primera" del manuscrito interrumpe el relato al final del f. $10 \mathrm{v}$ (cn la frase "Tamarys rreyna delas amazonas \& paso el Ryo" cfr. PCG, p. $220 \mathrm{~b}_{42}$ ) y lo retoma cn cl f. $14 \mathrm{r}$ ("cra ally adelantado delos rromanos..." cf. $P C G, 222 \mathrm{~b}_{48}$ ). Entre los ff. 10 y 14 hay tres folios en blanco, dos de los cuales fucron completados con un relato, ajeno a la $E f g$ o el $T R$, de la materia ausente, traducción del $D R H$ (l, xiii-xvi). Dudo si la laguna se debc a la pćrdida de un cuadcrno antes de que cl manuscrito fuese cncuademado en el siglo XVI o si el escriba había ya dejado los folios en blanco al percatarse de la ausencia del texto cn el cjemplar copiado.

${ }^{9}$ Incluyo aquí algunos datos sobre el códice. Comienza trunco por pérdida de su primer folio (f. 2/llr segín su numeración) en medio del prólogo del $T R$ : "su franqueza \& por su justiçia fueron nobles..." (cfr. D-ed $1: S_{21}$ ). El último folio que conscrva (279/CCLXXVIII), en muy mal cstado c incorporado al códice con el verso en el lugar del recto, termina en medio del capítulo dedicado a Enrique III, cn "... quatro galcas con quanto trayan a Barr [*meda c el rey mando] fazer dellas lo que fue su mer [*ced. Otrosi ese año se pa]saron de Portogal a Ca[*stilla Martin Vasquez]" (cfr. D-ed 2 : $107_{2426}$ ). También carece de su folio CCXXXI/231. Presenta al menos dos manos distintas. La primcra (ff. 2/II-76/LXXVI) dejó espacios de tres líneas para iniciales que no se añadieron. A partir del f. 77/LLXXII, y hasta el final de la crónica, desaparecen los cspacios para iniciales y el tipo de letra es distinto. Antepuesta al códice hay una nota mecanografiada que reproduzco parcialmente: - ${ }^{-[. . .]}$ Posiblemente este magnifico códice figuró en la biblioteca del conde de Haro, cuyo catálogo estudió tan diligentemente el Sr. Paz y Melia en la Revista de Archivos, Bibliotecas y Museos (tercera época, tomos l a XX) y de ahi pasó a la de su nieto Dn. Juan Fernandez de Velasco, insigne diplomático, y autor de las "Obscrvaciones del Prete Jacopin", tan encomiadas por Menéndez Pclayo". Una nota en portugués en el margen inferior del recto del primer folio conservado advicrte que el libro pertenece a la biblioteca "Do principe de Candea" y de la misma mano es la nota, tambićn en portugués, que aparece en una hoja de vuelta afiadida al final del códice: "1607. Fste famosisimo copioso e exquisito liuro e aBreujador de lodas as jstorias de España he de Jorge Roiz Dacosta o uuco $\mathrm{cm}$ Madrid o dito ano por ser de a liuvraria do condestable Joao Fernandez Velasco deu por elle hum dobrao en maio Jorge Roi da Costa. Foi este condestable o maior humanista $E$ dos mais bem entendidos sennores que ouue em nossos tempos. Requiscat in pace". El códice constaba en el catálogo de la Dolphin Book Co., Ltd. (Oxford), pero a principios de los noventa fue adquirido para la Biblioteca de la Universidad de Birmingham por el profesor Derek Lomax.

${ }^{10}$ Adapto la sigla propuesta por Catalán en su útimo trabajo (2005: 646, n. 26), donde obscrva la identidad del manuscrito cn su sección final con B, aunque no la que prescnta en sus primeros capítulos con D. El ms. es una copia cuidadosa por una única mano que cmpleó tinta roja para
} 
ta 1430", sección que hasta ahora se pensaba exclusiva del manuscrito fragmentario B y que Catalán (1966: 84-89 [=1992: 257-62]) consideraba una adición al texto de la crónica original. Asimismo, en los primeros capítulos de la historia goda $\mathbf{C}$ presenta el mismo peculiar comportamiento, ya mencionado, de $D$ al interpolar un texto de fuente desconocida al final del capítulo de las amazonas y ofrecer en los diez capítulos siguientes el texto del $T R$ y no el de ${ }^{*} \mathbf{L}$ ' $[<V V]$ como hacen el resto de los testimonios de la $E A$ (D-ed 1 : caps. xvi-xxv, $\left.28_{14}-429\right)^{11}$. Esta sustitución pudiera ser obra de un compilador que quiso completar con el $T R$ una laguna en el ejemplar de la $E A$ del que proceden $\mathbf{C}$ y $\star \mathbf{D}$ (ejemplar de la $E A$ sobre el que después de 1455 se realizó la refundición testimoniada por $D$ ). Dicha variante no se corresponde, por tanto, con la $E A$ refundida después de 1455 sino que es propia de la familia de $\mathrm{C}$ y *D', a la que, por otro lado, no es posible asignar con certeza a B, ya que el manuscrito está falto de todo el relato histórico hasta Frucla II y su final coincidente con $\mathbf{C}$ se encontraba también, como en seguida veremos, en el prototipo de la $E B$.

La principal novedad a esta lista, sin embargo, la aporta A, manuscrito copiado en 1545 por encargo de la duquesa de Alba a partir de un ejemplar perdido de la $E B^{12}$. Catalán (1966: 33 y n. 88 [ $=1992: 233$ y n. 9]) dio noticia del manuscrito, pero al no conocer

calderones, tibulos de capítulos e iniciales. Antepuestas al texto hay siete hojas de respeto añadidas por el encuadernador (siglo XVII). En ta ủitima de ellas se lee la siguicnte nota del siglo XVII: "Este Libro de la Historia de los Reyes de España escrito por el Arcobispo de Toledo D. Rodrigo Ximenez y adiccionado desde que acawo su Historia con la Conquista de Cordova fuc de Marques de Tarifa quien tubo otro escrito de la propia letra que se halla en la Bibliothoca de la Cartuxa de Serilla y en su principio Certifica el P. Fr Diego de Guelvas Prior que fue como lo dio dicho Marques con toda su Libreria y la fecha es $1^{\circ}$ de Heri de 1628. Acaua dicho Libro de la Cartuja en la vida del Rey Dn. Fernado el $4^{\circ}$ era 1348. El Presente sigue la vida de 6 Reyes D. Alfonso cl ultimo, D. Pedro, D. Henrique $2^{\circ}$, D. Juan el $1^{\circ}$, D. Henrrique el $3^{\circ}$ y D. Juan el $2^{\circ}$ hasta cl año 1430 , en que se acaua y siempre que habla dize este año por el de 1430 , donde se dectuze fue cn el que sc escrivio y en el vivia su author". La ficha de la Biblioteca de la Universidad de Sevilla rocoge cl contenido de esta nota y aúade que el libro "debió ser luego de D. Josć Bueno (léesc "Bueno" en los mingencs del índice)". El manuscrito ofrece abundantes anotaciones marginales contcmporáneas y posteriores a la copia Como detalle que pudiera ser significativo hay que serialar la presencia de dos asteriscos en el punto exacto del capitulo dedicado a Enrique III cn que finaliza el ms. S y comienza su baguns el ms. A de la $E B$, es decir, tras "que traian bastimento e venjan de Genoun" (C, f. 201r), lo que indica que el anotador conocía alguno de dichos manuscrilos u otro similar.

"Vid. Catalán 1966: 38-39 [=1992: 237]. El texto interpolado en el capitulo sobre las amazonas puede leerse en D-ed, $26_{22}-27_{10}$ desde "E asy mesmo dize la estoria" hasta "omes nengunos entre sy". Hacia el final de ese mismo capitulo $D$ y $C$ abandonan a * $L$ ' tras "morando alli pararon mientes...", y empalman con el $T R$ "...e vieron que cada dia eran pocas" (D-ed, $\left.28_{13-14}\right)$. Bi, F y $\mathbf{L}$ prosiguen ficles a ${ }^{2} \mathrm{~L}$ ": "pararon mientes c uicron commo menguauan sus fijas..." (Bi, f. $9 \mathrm{v}$ ).

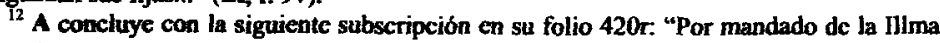
señora ta duquesa / de Alua Marquesa de Coria Etcetera Mi scrnora / Este libro scriujo Joannes de Canal clericus en Tala" y en el verso del mismo folio se añade la fecha: "Anno Domini Millesimo Quingentesimo / Quadragesimo Quinto / En Tala Arrabal dela villa de / Saluatierra de Tormes / Que es del Illustrisimo señor duque / De Alua Marques de Coria mi setior". 
de él más que sus líneas finales, lo asignó erróneamente a la $E A$ creyéndolo copia de B en vista de que concluye con la "Adición de Moguer". En realidad, A es un ejemplar de la $E B$, pues su distribución por fuentes coincide punto por punto con la observada por Catalán en dicho modelo cronístico ${ }^{13}$. El manuscrito está íntimamente emparentado con $\mathbf{S}$, siendo probablemente ambos copias independientes del mismo ejemplar ${ }^{14}$. Este parentesco es fácil de reconocer. En el capítulo dedicado a Enrique III, en el mismo punto que finaliza el texto de $\mathbf{S}$ con las palabras "con siete de Portugal que trayan bastimiento y venian de Genoa" (S, f. 283v cfr. D-ed 2 10719), el copista de A inserta la siguiente nota: "Aqui faltauan dos hojas queda esto en blanco para que se escriuan de otra historia como esta" (A, f. 415v), tras lo que efectivamente deja en blanco el resto de ese verso, el folio 416 y el recto y la mitad del verso del folio 417 , donde retoma el texto de su antígrafo en medio de una noticia de octubre de 1407 , primer año de Juan II, a partir de "[*Ortexi]car \& Audita y alas Cueuas y otros lugares todo esto por pleitesia que sc fuessen los moros en saluo y ansi los gano y puso su bastimiento..." (cf. B, f. 133v; C, f. 201v), hasta concluir con cl . relato de la conquista de Antequera y las noticias moguerienses que hasta ahora se creían exclusivas de la $E A$ adicionada en 1430 . A concluye en su folio $420 \mathrm{r}$ con "... todo esto ordeno la dicha doña

${ }^{13}$ Catalán, cn rcalidad, hubo de reconstruir el texto de la $E B$, al no contar más que con dos testimonios incompletos del texto: $\mathbf{S}$ (trunco por el comicnzo) y $\mathbf{T}$ (trunco por el final).

${ }^{14}$ Tambićn es estrecho su parentesco con $T$ en el fragmento conservado de dicho manuscrito. En la sección inicjal de la crónica, ausente en $\mathbf{S}$ por pćrdida de sus folios iniciales, A y $\mathbf{T}$ coinciden en omitir el final del capitulo correspondiente a $D R H \mathrm{I}, \mathrm{v}$. T, sin embargo, se distancia de $\mathbf{A}$ en esa misma sección al incluir la traducción de la "divisio orbis tripartita" antes del capítulo correspondiente a $D R H I$, ii y no después como hacen $A$, la $E A$, el $T R$ y los mss. del $D R I I$ que incluyen el texto. Los tres manuscrilos coinciden también en una seric de omisiones respecto al texto de las fucntes. Omiten, por ejemplo, desde el final del capítulo correspondiente a $D R I I I I$, i hasta cl principio de II, iii; los tres (S, f. 32v, igual en A y en T) cnlazan sin solución de continuidad "el qual dicho desviamijento les duro fasta el terçero conçilio de Tolcdo scgund dicho es c con aquella loca cregia" $(<D R H, \mathrm{II}$, i) con " $\mathrm{El}$ emperador Graçiano que començo a Reynar cra de ccc lxxxx i año despues que murio su tio" $(<D R H$, II, iii). Otra laguna común abarca cl final del capítulo dedicado al año segundo de Ordoño I y la mayor parte del capitulo siguientc (se omite, por tanto, lo correspondiente a Ded $1: 293_{15}-294_{29}$, desde "Ios de Sevilla salicron a ellos" hasta "Muça fuyo entonçe del campo con tres lançadas"). El escriba de A vuelve a indicar el texto ausente: "Aqui falta vna hoja del tercero año del rei Ordoño si no esto poco que se sigue" (A, f. 160r), mientras que $S$ y $T$ cmpalman el tex to sin solución de continuidad. No he comparado en detalle los textos de $\mathbf{A}$ S y T pero no creo que ninguno de los dos últimos sean copia directa del primero. $\mathbf{A}$, sin embargo, parece encontrarse más cercano al prototipo de la $E B$ pues conscrva mejores lecturas que los otros dos manuscritos. Por ejemplo, en cl capítulo del "laudes Hispaniae" S y T coinciden en un salto de igual a igual en la oración: "estos godos tobieron la Francia delos godos que es la provinçia de Narbona e avian otrosi en Africa vna provinçia (S, f. 77r igual en T) mientras que A (f. 113v) ofrece la lección completa: “...que es la provincia de Narbona con Rutherno y Alua \& Viuar, tres cibdades que enel tiempo delos godos pertenescian ala prouincia de Narbona $e$ avian otrosi... ("id cst Narbonensis prouinciam cum Ruthemo, Alba et Viuario ciuitatibus, que Gothorum tempore ad Nardonensem prouinciam pertinebant", DRH, III, xxi, cd. Fernández Valerde 1988: $105_{30-33}$ ). 
Francisca muger del dicho Micergilio. Laus Deo", el mismo final que ofrecen $B$ (f. 135r) y $C$ (f. 203r) de la $E A$. El ejemplar copiado en 1545 era, por tanto, un representante de la $E B$ que conservaba sus folios finales con la "Adición de Moguer" pero al que se le habían desprendido los folios inmediatamente anteriores. El escriba de A se apercibió de la laguna e incluso de su extensión gracias, probablemente, a los saltos que encontró en la numeración de los folios del ejemplar copiado. El copista de S, por su parte, reprodujo el mismo ejemplar, (o una copia del mismo cjemplar), pero concluyó la crónica en Enrique III, bien porque el antigrafo hubiera ya perdido sus últimos folios bien porque prefiriera no incluir la materia final sobre el reinado de Juan II dado su carácter fragmentario ${ }^{15}$.

Como resumen de todo lo anterior, el cuadro siguiente no prctende ser un stemma riguroso de la tradición textual de la crónica, sino ofrecer una representación gráfica de las correspondencias señaladas entre sus testimonios ${ }^{16}$.

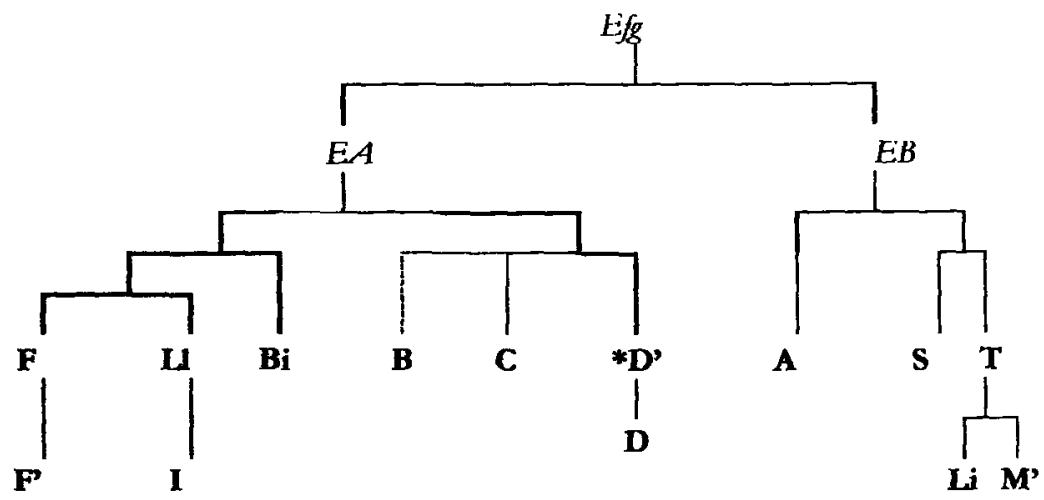

${ }^{15}$ A y S presentan idéntico comportamiento ante otra laguna del antigrafo común en el capitulo dedicado a Alfonso XI. Tras copiar "...al puento de Cebta y mataron mill y quinien[f. 395v] los moros" el copista de A advierte: "Aqui faltaua vna hoja. Queda esto cn blanco en que se cecriua de otro tibro que la tenga", tras lo que deja en blanco cl resto de ese verso, el recto del folio siguiente y sigue en el f. 396v: "los moros y tomaron la vianda que trayan delo qual..." (es decir, falta el texto correspondiente a D-ed $2: 61_{25}-63_{4}$ ). S, por su parte no se da cuenta del texto ausente y prosigue sin solución de continuidad, eliminando el scgundo "los moros", que considera un enror por repetición: "mataron mill c qujnicntos moros c tomaron la vianda que trayan delo qual..." (S, f. 281r).

${ }^{16}$ Sobre todo, es dudosa la posición del manuscrito B, trunco por el comienzo. Igualmente no es posible establecer si los mss. de la $E B$ derivan de la misma rama que $C, D$ (y $B$ ) de la $E A$ por las razones que se exponen en nuestro apartado 6 . No incluyo aqui los manuscritos en los que el texto de la Efg se encuentra mezclado con otras tradicioncs (vid. la nota 5). 


\section{FUENTES DE LA ESTORIA AMPLIA Y LA ESTORIA BREVE}

Una de las cuestiones que Diego Catalán dejó por responder en sus estudios sobre las $E f g$ fue la de la relación entre la $E A$ y la $E B$. Si bien la presencia en los dos textos de las mismas fuentes estructurales ( ${ }^{2} L$ ' y $T R$ ) y el hecho de que ambos concluyan con el mismo "Seguimiento del Toledano" indican un parentesco claro entre ellos, no resulta ciertamente fácil dilucidar el carácter exacto de la vinculación ni el del proceso de elaboración que los dio a la luz. Creo, sin embargo, que esta complejidad es sólo aparente y que una lectura atenta de la propia descripción realizada por Catalán (1966: 33-51 [=1992: 235-47]) de la estructura de las dos Estorias permite establecer de manera bastante concluyente la precedencia, cronológica y elaborativa, del estadio compilatorio correspondiente a la $E A$ respecto al de la $E B$. En la descripción del contenido de las dos Estorias que aquí ofrezco, deudora de la de Catalán aunque matizada con mis propias observaciones, he preferido dividir el relato histórico (desde el prólogo del arzobispo hasta el reinado de Juan $\Pi$ ) en 5 secciones, atendiendo a la convergencia o divergencia en el uso que respectivamente hacen de las fuentes.

\section{Prólogo, pobladores de España y 3 primeros capítulos de la historia goda}

Tanto la $E A$ como la $E B$ abren sus respectivos relatos con la introducción del arzobispo don Rodrigo y los capitulos relativos a la población de España según el TR. Al final de dicha narración, la $E B$ interpola (Interpolación [A]) el final del prólogo y los primeros 22 capítulos de la Versión primitiva de la $E E(V P)$ para volver luego a concordar con la $E A$ y ofrecer los tres primeros capítulos de la historia goda según * $\mathbf{L}^{\prime}[<V V]^{17}$.

\footnotetext{
${ }^{17}$ El interpolador anuncia: "Non embargante la primera scriptura en el comienço deste libro por tal de venir mas derecha en conosçimiento de las gentes que primera mente poblaron en España y delos fechos de Hercoles, començamos eneste capitulo que fabla del glorioso rey don Alfonso de Castilla el qual comiença segund se sigue" (T, f. 8v, igual en A). Al llegar al final de la interpolación enlaza con el inicio de la historia goda: "Estas cosas todas avernos traido sumaria mente alas difinjir por Razon que nucstra yntinçion fue de fablar enesta estoria primeramente de Ercoles y el Rey Ispan, que como conquistaron e fueron vençedores en España, c despućs ellos tio e sobrino poblaron comunal mente todo lo mas de España; y eso mjsmo Pirus yerno del Rey Ispan poblo asus vilas çibdades y castillos segund aquj es contado; des ende avemos fablado delas otras gentes que después destos vinjeron e conqujstaron a España fasta que el emperador Anjbal destruyo a Siguença e paso conquistar enla Italia. $E$ agora fablaremos delos godos que conquistaron e ganaron a España, que omes fueron et onde vinjeron" (S, f. 21 r, igual en A y T).
} 


\section{Reyes godos desde "Tanauso" y primeros reyes asturianos}

Concluidos los tres primeros capitulos de la historia goda, la $E B$ cambia de fuente para seguir al $T R$ hasta el final del reinado de Alfonso II. El relato ofrece, sin embargo, varias interpolaciones procedentes de la obra alfonsí, incluyendo la maldición al conde don Julián en el capítulo de la pérdida de España y una reelaboración del relato de la batalla de Roncesvalles (Conjunto de interpolaciones [B]). Por su parte, la EA se mantendrá fiel durante toda esta sección y la primera parte de la siguiente al texto de ${ }^{*} \mathbf{L}$, $[<V V+V E+V C]$, con la única excepción de la peculiar variante, ya señalada, que ofrece la familia de $\mathbf{C}$ y $\mathbf{D}$ en los primeros capitulos de la historia goda.

\section{II $^{18}$ \\ III. Desde el año $14^{\circ}$ de Alfonso II hasta el año $16^{\circ}$ de Vermudo}

Llegada al último capítulo que el arzobispo dedica al reinado de Alfonso II, la $E B$ inesperadamente abandona el $T R$ y vuelve atrás en el tiempo para coincidir con la $E A$ a partir del año $14^{\circ}$ de Alfonso II (recordemos que la $E A$ sigue reproduciendo el texto de * $L$, manuscrito que a estas alturas es representante de la $V C$ ), aunque omitiendo el capítulo sobre Roncesvalles, que ya había interpolado antes $^{19}$. Con este cambio de fuente el indudable propósito del compilador de la $E B$ es el de ofrecer a sus lectores la gesta de Bernardo del Carpio y, más adelante, la de los Infantes de Salas ${ }^{20}$. Ambos modelos cronísticos marchan de acuerdo en toda esta Sección III, coincidiendo en una serie de cambios de fuente, aunque el interés

\footnotetext{
${ }^{18}$ En esta sección perdemos el testimonio de $\mathrm{L}$ a partir del año $38^{\circ}$ de Alfonso II, pues el manuscrito finaliza trunco en ese punto. Es presumible que siguiera hermanado con ' $L$ " ofreciendo el texto de la $V C$ hasta su finalización posiblemente al final de la historia asturleonesa.

19 En su Sección mi $(E A=E B)$ la $E B$ también omite el final del primer capínlo de los dedicados al aho $31^{\circ}$ de Alfonso II relativo a les supuestas conquistas españolas de Cartomagno y el origen del camino de Santiago, sin duda, por haber tratado ya csa materia scgún la versión del TR. El capitulo termina "\& lo al dezid que chufan \& no es assi. Agora dexa la historia de fablar desto y toma a contar delos moros" (A, f. 146r, idéntico en S y T). Falta, por tanto, lodo lo correspondiente a D-ed 1 : 272 $2_{\mathrm{R}}$-74.

${ }^{20}$ Para realizar este cambio de fuente la $E B$ omite la noticia de la muerte de Alfonso II al final del último capitulo que el TR dedica a dicho rey $(D R H, I V$, xii) y, en su lugar, vuelve a anunciar, como ya habia hecto cn la Sección r: "Cornmo quier que non avemos fablado de don Bernaldo del Carpio cerca dela prision del conde San Dias su padre por fablar en estos fechos del Rey don Alfonso, conbicne nos tomar ala estoria enel catorzeno año del Rey don Alfonso el casto a fablar de Yxen amjramomeljn e dela prision del conde don Sant Dias padre de don Bernardo" (S, f. 97v; igual en A y T). A mi juicio, la intervención asegura la identidad entre el responsable de este cambio de fuente y cl interpolador que añadió la materia sobre los orígenes de España según la $V P$.
} 
del compilador de la $E B$ por la épica se vuelve a poner de manifiesto cuando introduce, en un momento en que la fuente de ambas crónicas es el $T R$, varios capítulos relativos a Fernán González y Garci Fernández, desconocidos de la $E A$ y procedentes nuevamente de la $V P(\text { Interpolación }[C])^{21}$. Por lo demás, la distribución de las fuentes de ambas Estorias en esta Sección III cs la siguiente ${ }^{22}$ :

a) Desde el año $14^{\circ}$ de Alfonso II hasta el año $4^{\circ}$ de Alfonso $11 \mathrm{ll}$ ambas redacciones ofrecen el texto de ${ }^{*} L$ ', representante aquí de la $V C$ (D-ed $_{1}, 263_{20}-301_{15}$. cfr. $V C$, caps. L-LXXXV, 453-524).

b) En el año $4^{\circ}$ de Alfonso III las dos redacciones abandonan a * $L$, [ $<V C]$ para narrar según el $T R$ el resto del reinado de Alfonso III (cfr. $D R H, \mathrm{IV}, \mathrm{xV}-\mathrm{xx}$ ) y los reinados posteriores hasta el de Ramiro III (D-ed $1,30 I_{15}-326_{22} c f r$. DRH, IV.xv-V.xii). Es en esta sección, tras el capítulo correspondiente a $D R H, \mathrm{~V}$.ii, (D-ed, $\mathrm{p}$. $314_{35}$ ), donde la $E B$ interpola el relato de la $V P$ sobre Femán González y Garci Femández.

c) A partir del primer capítulo dedicado al reinado de Vermudo II-y durante los dos capítulos siguientes ambas redacciones ofrecen una laboriosa colación de los textos del $T R \mathrm{y}^{{ }^{*}} \mathbf{L}^{\prime}[<V C]$ (D-ed, $326_{26}-332_{3}$ cf. $D R H, \mathrm{~V}$, xiii-xv y $C V R, \mathrm{~V}$, i-iii).

d) Durante los diez capitulos siguientes la $E A$ y la $E B$ abandonan completamente el $T R$ para narrar según ${ }^{2} L,[<V C]$ la traición y muerte de los siete Infantes de Salas, los acontecimientos ocurridos durante los años quinto a décimo del reinado de Vermudo II y la venganza de Mudarra (D-ed 1 : $332_{6}-350_{5} \mathrm{cf}$. $C V R$, V, vi-xx). En el siguiente capítulo (D-ed 1 , pp. $350_{9}-351_{22}$ ), dedicado a la batalla de Calatañazor (año $15^{\circ}$ de Bermudo II), ambas Estorias vuelven a ofrecer la mezcla de ${ }^{*} \mathbf{L}^{\prime}[<V C]$ y $T R$.

${ }^{21}$ Catalán (1966: 44-45 [=1992: 242-43]) scñala que el rclato sobre Fernán González interpolado por la $E B$ cs el propio de la $V P$ (que llama aquí "versión concisa") y es similar, por tanto, al que ofrece la $P C G$ en sus capítulos 684-96 (390-99), excepto por la omisión del capitulo correspondiente al segundo año de Ramiro II (PCG, cap. 685). La VP (de ta que procede la Versión amplificada que nos ofrece la $P C G$ ) sitúa los hechos de la vida de Fernán González, desde su alzamiento como conde de Castilla hasta la derrota del conde de Tolosa, en los años $1^{\circ}$ a $8^{\circ}$ del rey Ramiro II. En contraste, la $V C$ realiza una reorganización completa de todo este material, trasladando, por cjemplo, la narración del alzamiento del conde ( $P C G$, cap. 684, p. 390a) del año $1^{\circ}$ al $15^{\circ}$ de Ramiro Il y el episodio del sitio de Caraço ( $P C G$, caps. 687-88, pp. 391b-392a) del año $3^{\circ}$ de Ramiro II al año $2^{\circ}$ de Ordoño III (Catalán 1997: 397-98).

${ }^{22}$ Ofrezco las referencias al texto de la $V C$ en la edición de Fernández Ordóñez (1993) y, en la materia posterior al reinado de Ordoño II, las correspondientes a la edición de Ruiz Ascnsio et al (1991) de la Crónica de veinte reyes (citada CVR). Como sabemos, esta última crónica no hace sino reproducir el texto de la $V C$ a partir del reinado de Fruela II (vid. Fernández Ordóñez 1993: 65-114). 
IV. Desde el final de Vermudo II hasta el comienzo de los reyes de Aragón

Tras las primeras líneas del capítulo dedicado al año $16^{\circ}$ de Vermudo II (D-ed, $351_{30} c f r$. $C V R, \mathrm{~V}$, xxii) la $E B$ enlaza definitivamente con el $T R$ (a partir del capitulo correspondiente a $D R H, \mathrm{~V}$, xvii) ${ }^{23}$. Mientras tanto la $E A$ completa el capítulo con cl texto de ${ }^{\star} \mathbf{L}$ ' $\left[\langle V C]\right.$ y continúa ofreciendo la combinación de $T R$ y ${ }^{*} \mathbf{L}$ ' $[<V C]$ durante siete capitulos más (D-ed, $351_{30}-371_{13}$ cfr. $D R H, \mathrm{~V}$, xvii-xxiii y $C V R$, V, xxiii-VII.iv).

\section{Desde la historia de los reyes de Aragón hasta el reinado de Juan II}

La $E A$ no enlaza definitivamente con el $T R$ (y con la $E B$ ) sino una vez llegada al capítulo dedicado a la elección de Ramiro el Monje de Aragón ${ }^{24}$. A partir de dicho punto, las dos Estorias reproducen el texto del $T R$, fuente frente a la que ambas presentan sólo una variante común significativa: una versión del asesinato de Sancho II por Bellido Dolfos similar al relato de procedencia épica que ofrecen las crónicas generales. Por lo demás, los respectivos comportamientos de la $E A$ y la $E B$ respecto al $T R$ en esta Sección V son un tanto distintos: la $E B$ es más fiel a la letra de la fuente, mientras que la $E A$ tiende a abreviarla e incluso a introducir alguna interpolación puntual. En cualquier caso, una vez completado el relato del arzobispo, ambas lo proseguirán con el mismo "Seguimiento del Toledano" compuesto de la Historia hasta 1288 dialogada, un resumen de la Crónica de Alfonso $X I$ y el Poema de Alfonso Onceno, los Anales sevillanos (hasta 1407) y, en cl caso de B, C $(E A)$ y A $(E B)$ con la "Adición de Moguer hasta 1430".

${ }^{23}$ La EB (ms. S, f. 155v) abandona definitivamente el texto de * $L$ tras "saco su hueste este Abdelmelic fijo de Almanqor fue a correr ticrra de xpistianos e Robo e mato quanto fallo" (cfr. Ded, $351_{30} c f r . C V R$, V.xxii). La siguiente frase "e vino a Lcon e deRibo los muros e las torres e quanto alli fallo de lo que dexo..." pertenece ya al $T R$ ( $\angle D R H, \mathrm{~V}$, x vii).

${ }^{24} \mathrm{La} E A$ enlaza definitivamente con el $T R$ a partir de "e los más c los mejores ordenaron que fuesen al moncsterio \& to sacasen dende a don Ramiro ..." (cfr. D-ed, $371_{13}$ ), correspondientc a $D R H, \mathrm{~V}$, xxiii. 


\section{RELACIÓN ENTRE LA EA Y LA EB}

Completada esta descripción que, aunque parezca mentira, simplifica la composición por fuentes de los dos modelos compilatorios, ofrezco al paciente lector las conclusiones que extraigo respecto a la relación entre ellos de acuerdo con las distintas secciones identificadas en los textos.

Como se puede observar, las dos Estorias difieren en las fuentes empleadas en sus respectivas Secciones II y IV, mientras que son casi idénticas en las Secciones I, III y V (es decir, aproximadamente un $60 \%$ del volumen textual). Dejando para más adelante el examen de la Sección $\mathrm{V}$, parece claro que al menos en las Secciones I y III, ambas Estorias remiten a un arquetipo común, con las dos fuentes principales ya combinadas ( $T R$ y ${ }^{*} \mathbf{L}$ '), respecto al cual la $E B$ innova añadiendo el matcrial de las citadas interpolaciones [A] y $\mathbf{~} \mathbf{C}]$. Es significativo que tanto el texto de $[\mathbf{A}]$ (prólogo y primeros 20 capitulos de la $E E$ ) como el de $|C|$ (historia de Fernán González) remitan a la una versión de la $E E$ (la $V P$ ) distinta a las que en esas secciones debía ofrecer * $L$ ', el manuscrito del que tan abundante uso hace la $E A$ y, en menor medida, la $E B{ }^{25}$ En efecto, si * $L$ ' era hermano de $L$ en toda su extensión, y no hay razones para pensar que no lo fuese, en el inicio de la crónica ofrecería la $V V$ y en la historia de los reyes astur-leoneses la $V C$. Todo indica, por tanto, que el formador de la $E B$ contó entre sus fuentes con una "Crónica general" distinta de * $\mathbf{L}$ ' y cuyo texto era representante, en toda su extensión, de la Versión primitiva de la $E E$.

Para determinar la precedencia cronológica entre la $E A$ y la $E B$ será entonces preciso establecer cuál de las dos familias se aparta del arquetipo común en los trechos del relato en que ambas no coinciden en las fuentes que respectivamente emplean (Secciones II y IV). También aquí la evidencia textual apunta a que es la $E A$ la que se mantiene fiel al arquetipo, mientras que la $E B$ se aleja de él para innovar por su cuenta.

\footnotetext{
${ }^{25}$ La VP de la Estoria de España fuc elaborada en los talleres alfonsies hacia 1270 y abarcaba, al menos desde el inicio de la historia hasta el final del reinado de Vermudo III. Frente a esta redacción, en la sección anterior al reinado del godo Eurico se agnupan los manuscritos de la Versión vulgar, entre los que destacan, aparte de los testimonios que nos ocupan ( ${ }^{\prime} L$ ' y $L$ ), $Y$, To y $Q$. Aunque las variantes más características de la $V V$ aparecen en la historia de los godos, Catalán (1997: 65-69) identifica una serie de variantes en los primeros capitulos de la $E E$, ninguna de las cuales es compartida por el texto interpolado por la $E B$, el cual en todo momento se alinea con los manuscritos de la $V P$ (entre otros, $\mathbf{E}_{1}, \mathrm{C}$ y $X$. .). Así, la EB coincide con la VP al ofrecer en tres capitulos (PCG, caps. 11, 12 y 13) la materia que la $V V$ reúnc en uno solo. La $E B$ tampoco traslada el texto sobre las tres razones

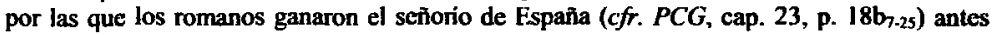
del capitulo sobre cl sctionío de los "almujces" ( $P C G$, cap. 11), como hace $L$ (f. 12v) siguiendo a la $V V$.
} 
Dicho carácter innovador de la $E B$ me parece evidente en la Sección n, pues aquí la $E B$ diverge de la $E A$ sólo después de haber reproducido los primeros tres capítulos sobre los orígenes de los godos según * $L$ ' $[<V V]$, es decir, tras haber coincidido previamente con la crónica hermana en su cambio de fuente anterior (del TR a *L) al iniciarse la historia goda. El compilador de la $E B$ reprodujo un texto idéntico al de la $E A$ en esos tres capitulos pero decidió abandonarlo para narrar la historia goda según el $T R$, bien porque su ejemplar presentase una laguna, bien porque, por alguna razón, prefiriese el relato del arzobispo (el cual, dicho sea de paso, es muy similar al alfonsí). A lo largo de su Sección II la EB incorpora material procedente de la $E E$ en varias ocasiones, incluyendo una serie de interpolaciones en los capítulos relativos a la pérdida de España y el episodio de la batalla de Roncesvalles (Conjunto de interpolaciones (B)). En los dos casos el texto se corresponde con el de las versiones Enmendada y Crítica de la $E E$, es decir, las mismas que ofrece la $E A$ en las secciones correspondientes del relato, por lo que la fuente debe ser \$L' 0 , lo que me parece más probable, la propia $E A$ (es decir, el mismo arquetipo con las fuentes ya combinadas que la $E B$ ya había reproducido en su Sección I).

Es posible argumentar que el mismo comportamiento se da al inicio de la Sección IV. Otra vez aqui parece ser la $E B$ la que abandona el arquetipo común, que había vuelto a seguir en su Sección II para inchuir la historia de Bernardo del Carpio y la de los Infantes de Salas, en vista de que su texto, una vez completada la gesta de los Infantes, no aportaba nada nuevo al relato del arzobispo. No olvidemos que cuando la $E B$ enlaza definitivamente con el $T R$, la $E A$ todavía tiene que ofrecernos siete capítulos más de la misma laboriosa colación de fuentes $\left({ }^{\star} L '[<V C]\right.$ y $T R$ ) que ambas Estorias presentaban en la Sección m. Asimismo, mientras que cabe alegar que el cambio de fuente en la $E B$ es deliberado-dado que la crónica ya nos ha ofrecido la venganza de Mudarra y un episodio que podía revestir cierto interés como el de Calatañazor- todo apunta a pensar que si la $E A$ deja de reproducir a * $L$ ' cuando se aproxima la conclusión de la historia de los reyes astur-leoneses es debido a la finalización o casi finalización de dicho manuscrito, representante de una crónica muy similar a la Crónica general Vulgata, cuyo relato termina con la muerte de Vermudo III de León ${ }^{26}$.

Con lo hasta ahora dicho se puede explicar la relación entre las dos versiones de la Estoria del fecho de los godos de acuerdo con el si-

\footnotetext{
${ }^{26}$ Segín Fernández Ordóntez (1997: 109-13 y 291-92), tanto *V (prototipo de la Crónica general Vulgata) como et prototipo común a $L$ y ${ }^{*} L^{\prime}$ remiten a un subarquetipo de la Versión crítica ("z") que terminaba con el reinado de Vermudo III. El otro subarquetipo ("y"), del que descienden cl manuscrito Ss y la CVR, llegaba hasta la muerte de Femando II de León.
} 
guiente modelo de transmisión textual. En un primer estadio (el comespondiente a la $E A$ ) se compilaron los textos de ${ }^{*} \mathbf{L}$ ', la "Historia gótica" del TR y las diversas fuentes que componen el "Seguimiento del Toledano" para producir un texto similar al de la $E A$. Por su parte, la $E B$ fue obra de otro compilador, u obra posterior del mismo compilador, quien procedió a combinar el texto del TR con el de la $E A$ (Secciones I y III y conjunto de interpolaciones [B]) y, menos extensamente, con el de una "Crónica general" representante en toda su extensión de la $V P$ (interpolaciones [A] y [C]). El evidente propósito de estos cambios de fuente por parte de la $E B$ fue ampliar el relato del romanceamiento con más datos sobre los origenes de España y los héroes de la epopeya leonesa y castellana (Bernardo del Carpio, Fernán González y los Infantes de Salas).

Me inclino, por tanto, a pensar que la $E B$ no emplea el texto de ${ }^{*} L$ ' directamente en sus secciones II y III sino a través de la $E A$ y, de la misma manera, creo que tampoco reproduce directamente el $T R$ en su Sección III (y, con menos certeza, en su Sección I) sino que copia su texto de la $E A^{27}$. En contraste, desde el inicio de su Sección IV y durante toda su Sección $\mathrm{V}$, hasta completar el relato del arzobispo, el compilador de la $E B$ pasó a seguir por su cuenta el $T R$ con total independencia de la transcripción que de dicha obra le ofrecía la Sección $\mathrm{v}$ de su ejemplar de la $E A$. Así lo sugiere el que en la $E B$ el cambio de prototipo se produzca antes y el que su texto no presente marca de sutura alguna en el punto en que cambia de fuente la $E A$, en la frontera entre las Secciones IV y V. Asimismo, la comparación entre los textos de las respectivas Secciones $V$ de las dos familias indica que ambas reproducen autónomamente la misma fuente, respecto a la que presentan comportamientos distintos: la $E A$ incorpora una serie de novedades respecto a la $E B$ y ofrece además una tendencia a abreviar, frente a la mayor fidelidad de la $E B$ al texto de los manuscritos "puros" del romanceamiento (H y E) y al propio $D R H^{28}$. Ofrezco los siguientes ejemplos, co-

${ }^{27}$ En sus respectivas Secciones ift, la $E A$ y la $E B$ comparten varias lagunas frente al texto del $T R$ cn los capitulos en que ambas versiones dependen del romanceamiento. También coinciden en añadir el año de la encarnación al de la cra que proporciona el arzobispo. El dato es importante a la hora de discernir la relación entre las dos versiones, pues la $E A$ mantendrá el mismo comportamiento en su Sección $\mathrm{V}$, mientras la $E B$ lo abandonará (vid. la nota 28). En cuanto a la Sección $I$, no estoy tan scguro de que la $E B$ reproduzca el $T R$ a través de la $E A$, ya que la lectura que del $T R$ ofrecen los mss. de la $E A$ es más defectuosa que la de la EB. Los primeros presentan, por ejemplo, una laguna que abarca la mayor parte del capítulo correspondiente a $D R H, \mathrm{I}, \mathrm{v}$, del quc solo transeriben las primeras lineas (cfr. D-ed, $16_{26-32}$ ). La $E B$ (representada aqui por A y $T$ ) ofrece el capitulo completo, a excepción de su sección final, donde se narra la instauración de Hispán como rey de España por Hércules, aunque la omisión pudiera ser deliberada ya que esta materia la $E B$ la narrará inmediatamente despućs según cl relato, más extenso, de la $E E$.

${ }^{28}$ Durante su Sección v la $E A$ innova respecto a la $E B$, el $T R$ y el $D R H$ al incluir al final de su último capitulo dedicado a Alfonso VII el traslado del cuerpo del empcrador por su hijo Sancho desde el puerto del Muradal hasta Toledo, noticia que en la $E B$ y cl $T R$ encabeza 
rrespondientes todos al relato del reinado de Alfonso VIII, para ilustrar este distinto comportamiento de ambas Estorias.

DRH

Et qui adhuc a mamilis nutricis paruuhus dependebat, etatis gracia fauorabilis, nature beneficio innocens, patris priuilegio amplectendus, futuronum indiciis obseruandus, quasi iam reus uel nocens ad mortem queritur, quasi non verum heres exhedatur, quasi non filius primogenitus imperatoris, qui deberat esse hers ex integro, a patrimonio effugatur. Quid mali fecit, qui loqui non poterat, qui nec statum sue infancie agnoscebat? (VII, xvii)
TR (ms. H)

$\mathrm{Y}$ el rrey, como quyer que fuese moco, ya dava a entender que tal seria despues que fucse grande, que bien dava a entender que hera de guardar \& de amar. Enpero seyendo tan pequetio que fascas mamava, a tal lo buscavan para lo matar, como sy fuese matador 0 rrobador, como sy fuese enemigo culpado. Tan poca verguenca le avian como sy no fuese nieto del enperador, heredero que devia de heredar todo entegra mente y echado de su patrimomio ique fue lo que fizo y lo que merescio?

(f. 194r)
$E B$ (ms, S)

Pero que el Rey don

Alfonso, commo qujer que fuesc moço, ya dava a entender que tal seria después que fuese grande, que bien dava a entender que hera de guardar e de amar. Pero seyendo tan pequeño tal lo buscaban como si fuese matador, como si fuese Robador, como si fuese enemigo, como si fuese culpado. E tan poca verguença le avian como si non fuese njeto del emperador don Alfonso e fijo del Rey don Sancho, ligitimo heredero; todo enteramente es corrido y echado del su patrimonjo ¿que fue lo que fizo, que fue lo que menesçio?

(ff. 207r-207v)
$E A$ (ms. Bi)

Avn seyendo tan pequeño lo buscauan para lo matar, commo sy fuese matador o rrobador 0 enemjgo \& commo sy non fuese njeto del enperador don Alfonso e heredero del Reyno. (f. 201v cf D-ed $444_{19-22}$ )

\footnotetext{
cl capitulo siguiente (Ded $:$ 436 $_{10-23} c f r . D R H$, VII, xi-xii). La $E A$ abre asi el siguiente capitulo con la subida al trono de Sancho III, cmpleando una fórmula similar a la que tan a menudo aparece en la $E E$ : "Muerto el Rey don Alfonso comenco a reinar su fijo don Sancho enla era...." (cfr. D-edi: 436 27). De la misma manera, la EA hace un capítulo con el relato de la llegada tardia de los reyes leonés y navarro a la lid de Alarcos y otro con el relativo al cerco almohade de Toledo (cfr. D-ed,: 45625-457 33 ), materia que ocupa sólo un capítulo en la $E B$, el $T R$ y el $D R H$ (VII, xxx). La $E A$ también ofrece varias interpolaciones al texto del TR desconocidas de la EB. Para empezar, sistcmáticamente añade cl año de la cncarnación a has referencias cronológicas que ofrecla el arzobispo (D-ed: $307_{26-27}, 311_{167} 316_{11}, 316_{27}$, $321_{\text {the }}, 322 n, 326_{33}$ ). Tambien innova en el capitulo relativo a ia conquista de Valencia por el Cid $(D R H, V I, x x v i i i)$ al informar de la fecha de la conquista y de los afios que el Cid gobernó la ciudad: "e ganola enel affo dela cncarmacion del Sefior de $M$ \& lxxxvij años, \& fue suya çinco antos fasta que fino ay" (cfr. D-ed, $412_{30-31}$ ) y concluir el capítulo remitiendo al leotor interesado en conocer mass sobre la vida del héroc a una Estoria del Cict. "segund todo esto \& mas corapljda mente cuenta enel su ljbro del Cid Ruy Diaz Canpeador por menudo" (cfr. Ded,$: 413_{1-3}$ ). Por último, al final del capítulo dedicado a la conquista de Cóndoba por Fernendo III (DRH, IX, xvi) la $E A$ ahiade: "e aquellos almogauares que primero entraron aujan nonbre el vno dellos Domjngo Colodro, e el otro auja nonbre Benjto de Baños. E por que tomaron primera mente e gararon la puerta del Colodro, que dizen oy dia, pusjeronle este nonbre, por el almogauar que dizen Domjingo Colodro. E aquel dia escurecio el sol todo al medjo dja" (cfr. D-ed, : $506_{28.37}$ ), una noticia desconocida de la $E B$, cl $T R$ y el $D R H$.
} 
In cuius morte fletus patrie, luctus inconsolabilis genitori, quia in ipsum tanquam in uite speculum contemplabatur, erat enim expectatio populorum; sic enim eum Dominus honestauerat ut etiam $\mathbf{a b}$ omnibus amaretur, et quod in adolescentibus non permitit, iam in ipso gracia dispensabat (VII, xxxvi)
\& enla su muerte fizo la tierra grand lloro \& grand llanto \& tomo grand quebranto su padre que lo tenia por espejo \& esfuerço dela su vida \& todos los pueblos avian enel esperança de anparo, ca asi lo cargara Dios de muchas bondades que todos lo amavan. \& lo que en los otros no cunple ny cifre la mancebia todo lo en este cunplia en graçia (f. 207r)
Quamis uero in oppidis et ciuitatibus sub uno degant principis regimine tamen a sue principio gentis et armorum usum et militaris dignitatis insignia habuerunt et militarem nomen sortiti sunt ab antiquo. Multa etiam contra Sarracenos et contra Christianos ipsi et eorum parentes decenter exercuerunt (VII, iii)
$\&$ hera gran derecho, ca ovieron de grandes tienpos derecho de fazer bien \& de aver vergueña \& de mantener grandes conpañas \& de ser costosos \& leales \& ovieron nonbre de cavalleros de antiguo, \& fueron omes que fizieron muchas cavallerias contra moros \& contra xristianos como debian (ff. 209r209v)
En la su muerte fizo la tierra grand lloro e gran llanto e tomo grand quebranto su padre, quelo tenia por espejo c esfuerço dela su vida, e todos los pueblos avian enel esperança de gran amparo, que ansi lo adomara Dios de muchas bondades que todos lo amavan. E lo que enlos otros no sufre la mancebia todo lo eneste cumplia la graçia de Dios (f. 219r)

Dela qual muerte fue grand quebranto al noble Rey don Alfonso su padre \& atodos los del Rey (f. $211 \mathrm{v}$ cfr. Ded $_{1}: 462$ 20-22)

$Y$ hera grand

derecho, que (f. 213r cfr. D-ed $: 465_{17}$ ) ovieron de grandes tiempos de derecho de fazer bien $e$ de aver verguença e de mantener grandes compañas e ser costosos e leales, e ovieron nombre de caballeros de antiguo. $E$ fueron hombres que fizieron muchas cosas e cavallerias contra moros e contra xpistianos como devian
Omitido en la $E A$ 
Junto a esta autonomía que entre sí mantienen en su Sección $\mathrm{V}$, no podemos olvidar las coincidencias fundamentales frente a la fuente que las dos versiones presentan en el capitulo relativo a la traición de Bellido Dolfos y en el propio "Seguimiento del Toledano" con el que ambas completan la narración del arzobispo. Mientras en lo que se refiere al relato común sobre Sancho II no es impensable que el fragmento se encontrase ya incorporado a la fuente común $^{29}$ (en cuyo caso habriamos de asumir que ambas Estorias emplearon el mismo ejemplar del TR), en el del "Seguimiento" me parece más probable que una vez llegado al final del $T R$, el compilador de la $E B$ lo reprodujera (aunque introduciendo, de nuevo, alguna interpolación) de su ejemplar de la $E A$.

\section{EL PROBLEMA DE LA FECHA DE LA EFG ORIGINAL}

Propongo, en definitiva, como conclusión al anterior examen de las fuentes de las $E f g$, que los manuscritos de la $E A$ son los representantes más fieles de la $E f g$ original compilada mediante la combinación de los textos de * $L$ ' y el TR y la adición de un "Seguimiento del Toledano", por lo que sería preciso, en rigor, abandonar la denominación de $E A$ y pasar directamente a llamar a dicho texto Estoria del fecho de los gados. A su vez, la $E B$ es, en realidad, un Toledano romanzado interpolado con la Estoria del fecho de los godos y la Versión primitiva de la EE o, si se prefiere, una Estoria del fecho de los godos interpolada con el Toledano romanzado y la Versión primitiva de la $E E$. Queda, sin embargo, establecer cuándo se elaboró esa $E f g$ original, una cuestión sobre la que, con la evidencia textual existente, no resulta posible pronunciarse con certeza por las razones que seguidamente expondré.

Dada la ausencia de evidencia alguna, interna o externa, de la identidad del compilador ni de la fecha que completó su labor, hemos de contentarnos con establecer un terminus post quem atendiendo a las últimas noticias recogidas en el texto, algo que siempre resulta comprometido en las obras cronísticas donde tan fre-

\footnotetext{
${ }^{29}$ Téngasc en cuenta que en los manuscritos "puros" del TR el relato del arzobispo sobre la muerte de Sancho II (DRH Vl.xviii) aparece ya interpolado con datos procedentes de las crónicas generales ( 60 del mismo cantar de gesta?), incluyendo el detalle de las espuclas que no llevaba el Cid Comparese la lección del original latino (ed. Fernández Valverde 1985: 199) con la del TR (el material nuevo va entre corchetes): "Salio un caballero delos de dentro dela villa a que dezian Vellido Dolfos [diziendo que venia a la merçed del mey] $\&$ andando el mey por el meal diole una lancada syn miedo ninguno. \& Ruy Dias Mio Çid fue en pos del conel sabor que avia de lo matar por su señor que lo avia muerto. \& ovicralo de alcançar enla cntrada dela puerta, sy no por la gran acucia que ovo Vellido Dolfos de se acoger a la villa de Camora. [\& dicen que Ruy Dias no llevava espuclas como que no se catava de tal trayçion pero que no es de creer que tal ome estudiese a caballo syn espuelas, mas de verdad las cosas pasan como Dios tiene por bien]" (H, f. 162r).
} 
cuente es la introducción posterior de adiciones actualizadoras al relato original. Dejando aparte $\mathbf{D}$, representante, como hemos dicho de una $E A$ refundida después de 1455 , como únicos testimonios del final de la crónica quedan los manuscritos $\mathrm{B}$ y $\mathrm{C}$ de la $E A$ $y$, aunque fragmentariamente, el manuscrito $A$ de la $E B$. En estos tres manuscritos los Anales sevillanos que concluyen la crónica culminan con una serie de noticias centradas en las actividades bélicas del regente don Fernando en la frontera durante los primeros años del reinado de Juan II. Se nos cuenta así la primera campaña andaluza en que se tomó Zahara y no se logró tomar Setenil (1407), la más afortunada incursión que culminó con la celebrada conquista de Antequera (1410) y un relato de carácter muy local que nos narra cómo, a la muerte de Pedro Portocarrero en 1430, el señorio de Moguer no recayó en sus hijas, sino en su hermana Francisca y su marido Micer Gilio Bocanegra.

Basándose en un salto en la continuidad analística entre la noticia de la salida del infante don Fernando de Sevilla en noviembre de 1407 y el relato de la conquista de Antequera en abril de 1410, Catalán (1966: 84-89 [=1992: 257-62]) considera que todas las noticias restantes (conquista de Antequera y sucesos moguerienses) son un añadido ("Adición de Moguer") introducido después de 1430 al cuerpo de la crónica ya formada ${ }^{30}$, para cuya composición propone las fechas de abril de 1407 y 1410 como respectivos términos post y ante quem. Hemos de recordar, sin embargo, que $\mathrm{Ca}$ talán sólo conoció de esa sección final del relato la lección del ms. B de la $E A$ y desconocía, por tanto, el testimonio de $\mathbf{C}$ y, sobre todo el de $A$ de la $E B$, manuscritos que hacen albergar dudas respecto al carácter añadido de la sección de 1410 a 1430 . Ante todo, estos manuscritos nos indican que la presencia de la "Adición" estaba más cxtendida en la tradición textual de la $E f g$ de lo que originalmente se pensaba ${ }^{31}$. Por lo pronto, dado el parentesco por el principio entre C y D, podemos asumir que la "Adición" se encontraba en el manuscrito fuente de la Refundición después de 1455. Más importante aún es que la presencia del texto en $\mathbf{A}$ lo vincula a la rama de la $E B$ y nos lleva, por tanto, a pensar, que si $S$ y $T$ no lo ofrecen es por sus finales defectuosos.

\footnotetext{
${ }^{30}$ El relato de la "Adición de Mogucr" no se encuentra asociado a los Anales sevillanas en los dos testimonios con que contamos de ellos como obra independientc a las Estorias del fecho de los godas: el "Suplemento" a la Crónica de Enrique III de Pero López de Ayala (vid. Rosell 1877: 245-46) y los Annales eclesiasticos y seculares de la muy noble y muy leal ciudad de Sevilla de Diego Ortiz de Zúñiga (1677: 283 y 284), donde son empleados muy abundantemente hasta el año 1407.

${ }^{31}$ A estos testimonios hay que sumar los de las crónicas de Pedro de Escavias y Diego de Valera, quienes conocieron una $E A$ que incluia las noticias postcriores a 1407 (vid. la nota 7).
} 
En vista de ello, parecería obligado que nos hubiésemos de contentar con la fecha de diciembre de 1430 (fecha de la última noticia mogueriense) como termimus post quem del arquetipo común a ambas Estorias (es decir, la compilación de la que últimamente se derivan todos los manuscritos conocidos). En efecto, en vista de que el final de $A$, representante de la $E B$, coincide con el de los dos únicos manuscritos completos de la $E A$, no hay evidencia verdaderamente concluyente que sostenga que las noticias de 1410 y 1430 (la "Adición de Moguer") sean una adición posterior a una crónica formada en 1407, a la luz de su presencia en dos modelos cronisticos que barajan de manera distinta las mismas fuentes principales $\left(T R y^{*} L^{\prime}\right)^{32}$. El esquema de transmisión simplificado sería el siguiente:

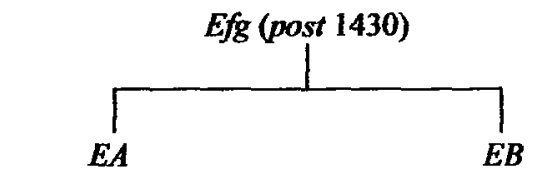

B Bi C D F L A S T

Con todo, nuestra hipótesis de que la $E B$ es una reelaboración posterior de la $E A$ impide descartar del todo la datación post 1407 y ante 1410 que Diego Catalán sugiere para la Efg original, pues nada impide que el compilador de la $E B$ hubiese empleado un manuscrito de una " $E A$ adicionada en 1430".

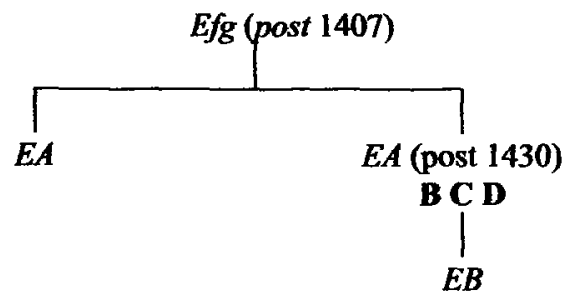

A S T

En este segundo supuesto se plantea, claro está, el problema de dónde situar en el stemma los manuscritos incompletos $\mathbf{B i}, \mathbf{F}$ y $\mathbf{~ L l}$ ¿Son representantes de la hipotética crónica original de 1407 o de la "versión adicionada" de 1430? La cuestión me parece irresoluble $y$, en ausencia de otros testimonios, la elaboración de un modelo

${ }^{32}$ Ello implicaria, por supuesto, que el final moguericnse se encontraba también en la rama de la que derivan el resto de los manuscritos de la EA, Bi, F y LL, truncos, como sabemos, de sus respectivos finales. 
definitivo de la transmisión de la crónica, así como la cuestión de si fue elaborada en 1407 o 1430 deben quedar aplazadas ${ }^{33}$.

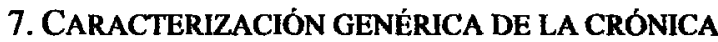

La Efg es un ejemplo tardío de "Crónica general", un género que representa la vida "tradicional" en los siglos XIV y XV de los textos compuestos por el taller historiográfico alfonsi durante los años $1270-82^{34}$. La labor del formador o de los formadores se limitó a reproducir y yuxtaponer fragmentos de crónicas anteriores sin incorporar material propio (excepto, tal vez, el final de la crónica y los arreglos que ligan las diversas fuentes del "Seguimiento del Toledano") ni, como suele ocurrir con las crónicas generales, dotar al discurso resultante de una nueva voz narrativa. Si la crónica aporta alguna novedad historiográfica es la de editar el texto alfonsí de acuerdo con el esquema histórico del $D R H$ de Rodrigo Jiménez de Rada, y la de presentarse ante el lector como una traducción (amplificada) de esa obra. La compilación da así testimonio del prestigio de don Rodrigo como máxima autoridad historiográfica de la época y podría incluso inscribirse en un renacimiento del modelo neogótico de historia de España del arzobispo de Toledo, observable desde finales del XIV y que alcanzaria su canonización definitiva en la segunda mitad del siglo XV de la mano de autores como Alfonso de Cartagena o Rodrigo Sánchez de Arévalo $^{35}$. Asimismo, por su indudable interés en las líneas maestras de la historia, la $E f g$ también se inserta en el género de las "sumas de crónicas" elaboradas en lengua romance durante la primera mitad del siglo XV. A diferencia de la Efg éstas eran obras personales pero cuyos autores también muestran una preferencia

${ }^{33}$ Un dato que pudiera ser significativo es que el ya citado Sumario de la EA (=BN 7404) realizado en cl siglo XVII termine con la muerte de Enrique III (25 de diciembre de 1406), lo que sugeriria que el manuscrito abreviado concluía en ese punto, o inmediatamente después, y que tal vez se tratasc, por tanto, de un ejemplar de la Efg desprovisto de las noticias posteriores a 1407. Personalmente, de los dos modelos de transmisión presentados me inclino más por el de un arquetipo posterior a 1430 (y anterior a 1455) o, en el caso del segundo modelo, por un arquetipo poco anterior a 1430, ya que la utilización diversa de, al menos, una misma fuente por las dos Estorias (cl $T R$ ) y la probabilidad de que se tratase del mismo cjemplar de la obra (dada la presencia cn ambas versiones del material épico relativo a la traición de Bellido Dolfos), sugieren una composición cercana cn el tiempo y en el espacio, tal vez el mismo agente compilador, y hacen poco probable la existencia de un intervalo de tiempo demasiado amplio critre ellas.

${ }^{34}$ De hecho, la obra fue durante mucho tiempo conocida como Cuarta crónica general, título con el que la habia bautizado Menéndez Pidal (1898: 97-98).

${ }^{35}$ Sobre el neogoticismo de la historiografia del siglo XV véanse los trabajos de Brian Tate (1970 y 1996) y el clásico de Maravall (1955) especialmente el capitulo "La tradición de la herencia goda" (315-58). 
por el ideal neogótico e invocan la autoridad del arzobispo don Rodrigo para enunciar su discurso (Jardin 2000).

En la formación de las dos versiones de la Estoria del fecho de los godos se observa, por tanto, una tensión entre dos propósitos compilatorios, correspondientes a dos tendencias genéricas de la historiografia castellana del siglo XV: por un lado, cl de ofrecer una visión de conjunto de la historia hispana de acuerdo con el modelo neogótico propio de las sumas de crónicas; por el otro, el de satisfacer la demanda del público lector por los atractivos contenidos de la épica que se hallan en las crónicas generales. El compilador logró llegar a un compromiso entre estos dos criterios y ofrecer, en un único volumen relativamente manejable, un relato completo de la historia de España, avalado por la autoridad del arzobispo don Rodrigo y complementado con versiones "cumplidas" de algunos de los episodios más populares de la epopeya, en concreto los relativos a Carlos Mainete, Bernardo del Carpio y los siete Infantes de Salas.

8. CONCLUSIONES: LA EFG Y EL OFICIO DE HISTORIAR EN LA ESPAÑA BAJOMEDIEVAL

Concluyo muy brevemente con las reflexiones prometidas al inicio de este trabajo. Más allá de consideraciones de carácter general, el anonimato del compilador de la $E f g$ y su estricta fidelidad a la letra de la fuente que en cada momento reproducía hacen que sea dificil precisar las circunstancias de composición de la obra y los criterios e intencionalidades que rigieron la selección y distribución de las fuentes ${ }^{36}$. En realidad, aparte de las intervenciones editoriales que implican la introducción de materiales épicos, a menudo no parece haber otro motivo para el empleo de una fuente u otra que el capricho del compilador o las propias limitaciones

\footnotetext{
${ }^{36}$ Con todo, en mi tesis doctoral (2004) me aventuraba a presentar una serie de conjeturas sobre las posibles circunstancias y de composición de la $E f g$ y su entorno ideológico. El evidente caricter sevillano de la $E A$, patente sobre todo en su empleo de fuentes como la Historia dialogada y los Anales antiguos sevillanos, me llevaba a proponet que el empleo de l cononica general ("L') pudiese haber estado motivado por el carácter más "sevillista" del relato alfonsi en secciones como la de la historia goda -piénsesc por cjermplo, en la "hitación de Bambe" incluida en la $E E$ y su referencia a la primacía histórica de la iglesia sevillana en ln época visigótica - frente al contenido del $T R$, necesariamente más parcial hacia Toledo como traducción directa que es del $D R H$. Asimismo, y aceptando que la crónica original datase de poco después de 1407, me preguntaba si la formación del texto no habria estado relacionada con las estancia en Sevilla del infante don Fernando, tío de Juan II y regente de Castilla, junto con Catalina de Lancaster, con ocasión de la campana contra el reino de Granada que dirigiria durante el último trimestre de 1407 (las últimas noticias incluidas en la supuesta Efg original se reficren a esta incursión). Las imprecisiones y el descuido en la labor compilatoria pudieran deberse a que la crónica hubiese sido claborada apresuradamente para serte presentada como obsequio al infante.
} 
materiales que le imponían sus fuentes. Asi, por ejemplo, parece claro que el hecho de ofrecer la historia de los reyes de Castilla según el $T R$ no obedece sino a la terminación del manuscrito * $\mathbf{L}$ ' al final de la historia de los reyes leoneses. Menos evidentes son los criterios del compilador de la $E A$ a la hora de utilizar el relato ${ }^{*} \mathbf{L}$ ' sobre los reyes godos, cuando el del $T R$ es bastante similar, o los que le llevaron a no ofrecer el final de la gesta de Bernardo el Carpio según ‘ $L$ ' (¿una laguna en el manuscrito?) o, menos aún, a realizar una meticulosa colación de fuentes ( ${ }^{\star} L '$ y $T R$ ) en ciertos capitulos de la historia leonesa. La impresión es, por tanto, que la crónica -y me refiero aquí a la $E A$ aunquc la afirmación es también válida para la $E B$ - fue obra de un agente compilador que fue aprovechando las pocas fuentes a su disposición para rellenar, a menudo con bastante tosquedad, sus lagunas respectivas y componer un relato completo e ininternumpido de la historia de España. Esta penuria compilatoria es un rasgo común a la mayoria de modelos de crónica general y puede ser, a mi juicio, efecto de la popularidad de este tipo de textos y, posiblemente, de la ampliación "hacia abajo." de la élite social a la que iban dirigidos. A lo largo del siglo XIV la historiografia romance, una vez libre del control directo de la corona, fue abandonando el ámbito estrictamente cortesano para difundirse en un entorno social cada vez más amplio, la nobleza grande y pequeña, el clero e incluso la burguesía urbana.

Me parece oportuno, sin embargo, observar que si bien en cuanto a los recursos materiales e intelectuales a su disposición los formadores de crónicas generales, y de la $E f g$ en concreto, están a años luz de la riqueza archivística y la sofisticación compilatoria de la $E E$ original, obra fruto de un proyecto patrocinado por la corona castellana y para cuya elaboración no se repararon dispendios, en cuanto a los principios metodológicos subyacentes a su trabajo las diferencias no son tan abismales. Al fin y al cabo, el compilador de la $E f g$ también realiza, dentro de un ámbito mucho más modesto y a una escala mucho menor, las mismas operaciones de "reproducir, reunir, construir, combinar y revisar" que Georges Martin (1991) identificaba en la composición de la $E E$. Dichas operaciones se derivan de una concepción de la labor del historiador entendida como esfuerzo por reunir textos dispersos y reconstruir las memorias fragmentarias que contienen con el fin de asegurar su pervivencia y transmisión, como nos recuerdan tantos prólogos de crónicas medievales.

En este sentido creo que el estudio de obras como la $E / g$ puede iluminar áreas dificiles de acceder dentro de la historiografia y de la cultura textual bajomedieval en general. Son testimonio, por ejemplo, del valor "crematístico" que para los agentes transmisores 
tenía el texto recibido. En una cultura manuscrita cualquier texto, y especialmente si estaba atribuido a un autor prestigioso o pertenecia a un género mayor como el historiográfico, debia ser un bien valioso, merecedor de ser conservado y transmitido independientemente del sesgo ideológico de sus contenidos. Al mismo tiempo, los compiladores son muy conscientes de que en el curso de su transmisión manuscrita los textos sufren un deterioro que es preciso paliar produciendo un texto de mayor calidad que el del ejemplar recibido. En esta labor los compiladores medievales se suelen comportar de manera muy similar a los filólogos modernos. Como ellos son adeptos a comparar textos, detectar en ellos coincidencias y variantes y reconstruir originales perdidos y más perfectos (de ahí la práctica de la colación que encontramos en la $E f g$ ). Por otro lado, dado que la historia es memoria, la misión del historiador es complementar el relato de la fuente con nuevas "razones", y aquí se incluyen la actualización hasta el presente del relato cronístico y su ampliación con noticias que pucdan encontrarse en otros textos o, a menudo, en las anotaciones en los márgenes del cjemplar reproducido. Todas estas prácticas, consustanciales a la labor del historiador medieval, se manifiestan en una contaminación constante $e$ indiscriminada entre los distintos modelos y, consecuentemente, en unos textos eminentemente "mixtos", o incluso "hibridos" y, lo que es más importante, en estado de constante transformación, pues cada agente transmisor es un autor potencial de variantes, interpolaciones y actualizaciones. En un artículo reciente Diego Catalán (2000) ha señalado estas características de los textos cronísticos y advertido del desafio que representan para la crítica textual, sobre todo a la hora de establecer con precisión los distintos estratos compilatorios subyacentes al texto recibido.

Creo que estas reflexiones son oportunas a la hora de juzgar las conclusiones presentadas en este trabajo. Si bien es posible aislar con cierta seguridad un estado sincrónico del texto de la $E f g$ que remonta a poco después de 1430 (el representado por los manuscritos completos de la $E A$ ), en lo que se refiere a los estratos compilatorios precedentes nos hemos de mover en la esfera de las hipótesis más o menos plausibles y nos vemos incapaces de establecer con certeza una fecha ante quem para la crónica original. Pero también es moverse en el terreno de la probabilidad atribuir a un único esfuerzo compilador la combinación de tres núcleos textuales: * $L$ ', el TR y las fuentes del "Seguimiento del Toledano". Aunque se trata de un modelo plausible, es resultado, en cierta medida, de aplicar la navaja de Ockam, y es evidente que la lógica combinatoria admitiría otros, cuya verificación o refutación, en ausencia de nuevos testimonios, nos llevaría a una discusión casi ad infinitum. Sería posi- 
ble, por ejemplo, que la combinación de * $L$ ' y $T R$ remitiese a una compilación anterior y que el "Seguimiento" se hubiese añadido años más tarde, o bien que el TR y el "Seguimiento" formasen un cuerpo textual previo a la combinación con ${ }^{*} \mathbf{L}$; por último, cabe también la posibilidad de que las distintas secciones del "Seguimiento" hubiesen sido añadidas durante distintas etapas sucesivas de la transmisión del texto.

Este fenómeno de la multiplicación de posibilidades a la hora de establecer los diferentes estadios en la transmisión de un texto cs común en las obras medievales pero parece mucho más acentuado en los textos cronísticos y, aunque en el caso de una obra relativamente secundaria como la $E f g$ tal vez no revista demasiada gravedad, sí constituye un aviso para aquellos navegantes embarcados en el estudio de otras crónicas de mayor entidad literaria e histórica, y me refiero especialmente a uno de los nudos gordianos que queda aún por desenredar dentro de la tradición historiográfica alfonsí: la historia de los reyes de Castilla de la Estoria de España.

\section{ABREVIATURAS DE LAS OBRAS CITADAS}

CA Crónica abreviada de Diego de Valera

CVR Crónica de veinte reyes

D-ed ${ }_{1} \quad$ Volumen 105 de la edición del marqués de la Fuensanta del Valle (1873)

D-ed 2 Volumen 106 de la edición del marqués de la Fuensanta del Valle (1873)

DRH De rebus Hispaniae sive Historia Gótica

Efg Estoria(s) del fecho de los godos

EA Estoria amplia del fecho de los godos

EB Estoria breve del fecho de los godos

EE Estoria de España

TR Toledano romanzado

VC Versión critica de la Estoria de España

VE Versión enmendada después de 1274 de la Estoria de España

VP Versión primitiva de la Estoria de España

VV Versión vulgar hasta Eurico de la Estoria de España

\section{OBRAS CITADAS}

Bustos, María del Mar, (2002), "Estoria del fecho de los godos", en Diccionario filológico de literatura medieval española. Textos y trans- 
misión, ed. Carlos Alvar y José Manuel Lucía Megias, Madrid, Castalia, pp. 476-87.

CAtalán, Diego, (1996), "El Toledano romanzado y las Estorias del fecho de los godos del siglo XV", en Estudios dedicados a James Homer Herriott, Madison, University of Wisconsin, pp. 9-102.

CATALÁN, Diego, (1992), La Estoria de España de Alfonso X: creación y evolución, Fuentes Cronisticas de la Historia de España, 5, Madrid, Fundación Menéndez Pidal, Universidad Autónoma de Madrid \& Gredos.

CATALÁn, Diego, (1997), De la silva textual al taller historiográfico alfonsí: códices, crónicas, versiones y cuadernos de trabajo, Fuentes Cronísticas de la Historia de España, 9, Madrid, Seminario Menéndez Pidal, Fundación Ramón Menéndez Pidal \& Universidad Autónoma de Madrid.

CataláN, Diego, (2000), "Monarquía aristocrática: Rodrigo en la Crónica de Castilla", en La historia alfonsi: el modelo y sus destinos (siglos XIII-XV), Collection de la Casa de Velázquez 68, ed. Georges Martin, Madrid, Casa de Velázquez, pp. 73-94.

Catalán, Diego y JEREZ, Enrique, (2005), "Rodericus" romanzado en los reinos de Aragón, Castilla y Navarra, Fuentes Cronisticas de la Historia de España, 10, Madrid, Fundación Menéndez Pidal.

Crespo, Juan Bautista, (2000), "La Estoria de España y las crónicas generales", en Alfonso $X$ el Sabio y las crónicas de España, ed. Inés Fernández Ordóñez, Valladolid, Universidad de Valladolid-Centro para la Edición de los Clásicos Españoles, pp. 83-132

FAULHABER et al., (1984), Bibliography of Old Spanish Texts, $3^{\text {rd }}$ edition, Madison, Hispanic Seminary of Medieval Studies.

FERNÁNDEZ ORDÓNEZ, Inés, ed., (1993), Versión crítica de la Estoria de España: edición y estudio desde Pelayo hasta Ordoño II, Fuentes Cronísticas de la Historia de España, 6, Madrid, Seminario Menéndez Pidal, Fundación Menéndez Pidal \& Universidad Autónoma de Madrid.

FERNÁNDEZ ORDóÑEZ, Inés, (2000), "La transmisión textual de la Estoria de España y de las principales Crónicas de ella derivadas", en Alfonso X el Sabio y las crónicas de España, ed. Inés Fernảndez Ordóñez, Valladolid, Universidad de Valladolid-Centro para la Edición de los Clásicos Españoles, pp. 219-64.

FERNÁNDEZ VAlVERDE, Juan, ed., (1987), Rodericii Ximenii de Rada, Opera omnia I: Historia de Rebus Hispaniae sive Historia gothica, Corpus Christianorum. Continuatio Mediaevalis, 72, Turnhout, Brepols.

Fuensanta del Valle, Marqués de la, (1893), Crónica de España del arzobispo Don Rodrigo Jiménez de Rada. Tradújola en castellano y la continuó hasta su tiempo Don Gonzalo de la Hinojosa y después un anónimo hasta el año de 1454. Bibli ${ }^{a}$ Nac. -Dd-179, Colección de documentos inéditos para la Historia de España, 105 \& 106, Madrid: Perales y Martinez; repr. Vaduz: Kraus Reprint, 1966. 
GaRCIA, Michel, ed., (1972), Repertorio de Príncipes de España y Obra poética de Pedro de Escavías, Instituto de Estudios Giennenses del C.S.I.C. \& Diputación Provincial de Jaćn.

GómEZ PÉrez, José, (1962), "La más antigua traducción de las Crónicas del Toledano", Hispania, 87: 357-71.

HiJANO VILLEGAS, Manuel, (2003), "Estoria del fecho de los godos: estudio y edición", 2 vols., tesis doctoral, University of Birmingham.

JARDIN, Jean Pierre, (2000), "El modelo alfonsí ante la revolución trastámara. Los sumarios de crónicas generales del siglo XV", en La historia alfonsi: el modelo y sus destinos (siglos XIII-XV). Seminario organizado por la casa de Velásquez (30 enero de 1995), ed. Georges Martin, Colección de la Casa de Velázquez 68, Madrid, Casa de Velázquez, pp. 141-56.

MARTIN, Georges, (1991), "Cinq opérations fondamentales de la compilation: l'exemple de l'Histoire d'Espagne (Étude segmentaire)", L'historioraphie médiévale en Europe, ed. Jean Philippe Genet, París, Éditions du CNRS, pp. 99-109.

MaRAVAlL, José Antonio, (1954), El concepto de España en la Edad Media, Madrid, Instituto de estudios políticos.

Mata Carriazo, Juan de, ed., (1941), Diego de Valera. Memorial de diversas hazañas. Crónica de Enrique IV, Madrid, Espasa Calpe.

MENÉndez PIDAL, Ramón, (1898), Crónicas Generales de España. Católogo de la Real Biblioteca. Manuscritos 1, Madrid, Sucesores de Rivadeneyra.

MEnÉndez PidAl, Ramón, ed., (1955), Primera crónica general de España que mandó componer Alfonso el Sabio y se continuaba bajo Sancho IV en 1289, 2 vols., Madrid, Gredos.

ORTIZ DE ZÚÑIGA, Diego, (1677), Annales eclesiasticos y seculares de la muy noble y muy leal ciudad de Seuilla, Madrid, Juan Garcia Infançon.

RODRIGUEZ DE CUENCA, Juan (1781), Sumario de los reyes de España por el Despensero mayor de la Reyna Doña Leonor, muger del Rey Don Juan el primero de Castilla, con las alteraciones y adiciones que posteriormente le hizo un anónimo, ed. Eugenio de Llaguno Amirola, Madrid, Antonio de Sancha (reimpr. facsímil con índices realizados por María Desamparados Pérez Boldo, Valencia, Anúbar, 1971).

Rosell, Cayetano, ed., (1875 \& 1877), Crónicas de los reyes de Castilla desde Alfonso el Sabio hasta los católicos don Fernando y doña Isabel, Biblioteca de Autores Españoles, 66 \& 68, Madrid, Rivadeneyra.

RuIZ ASENSIO, J. M. et al, (1991), Crónica de veinte reyes, Burgos, Ayuntamiento de Burgos.

SÁNCHEZ ALONSO, Benito, (1925), "Las versiones en romance de las crónicas del Toledano", en Homenaje ofrecido a Menéndez Pidal, I, Madrid, Librería y casa editorial Hernando, pp. 341-54.

TATE, Robert Brian, (1970), Ensayos sobre la historiografia peninsular del siglo $X V$, Madrid, Gredos.

TATE, Robert Brian, (1996), "The rewriting of the historical past: Hispania et Europa", en Historical Literature in Medieval Iberia, ed. 
Alan Deyermond, Papers of the Medieval Hispanic Research Seminar 2, London, Queen Mary and Westfield College, pp. 85-103.

VAlERA, Diego de, (1527), La Cronica de España abreviada por mandado de la catholica \& my poderosa Señora doña Isabel Reyna de Castilla \&cetera, Sevilla.

VAN DER WALT, Carol Ann, (1999), "A Critical Edition of the Historia Gótica, a Vernacular Translation of Rodrigo Ximénez de Rada's De Rebus Hispaniae", tesis doctoral, University of Birmingham. 
8003

RESUMEN: La Estoria del fecho de los godos es una crónica formada a principios del siglo XV mediante la combinación de diversas fuentes historiográficas, incluyendo materiales pertenecientes a la tradición de la Estoria de España alfonsí y una traducción del De rebus Hispaniae de Rodrigo Jiménez de Rada conocida como Toledano romanzado. Tras pasar revista a los testimonios de la obra, se procede a su clasificación en familias y subfamilias, para luego describir la estructura compositiva de las dos principales versiones del texto (Estoria amplia y Estoria breve). Dicho estudio permite proponer una serie de hipótesis sobre la vinculación entre las dos versiones y la fecha de composición de su arquetipo común. Como conclusión, se comentan los problemas metodológicos que para la critica textual plantea esta compilación historiográfica, y sus implicaciones para el estudio de las otras "crónicas generales de España" de la época bajomedieval

Palabras Clave: Alfonso $\mathrm{X}$, crítica textual, crónica, crónicas generales de España, De rebus Hispaniae, Estoria de España, historiografia, Esto-. ria del fecho de los godos, Estoria amplia del fecho de los godos, Estoria breve del fecho de los godos, Jiménez de Rada, siglo XV, Toledano romanzado.

ABSTRACT: The Estoria del fecho de los godos is an early fifteenthcentury chronicle compiled from a variety of sources, including materials belonging to the tradition of King Alfonso X's Estoria de España as well as a translation of Rodrigo Jiménez de Rada's De rebus Hispaniae known as Toledano romanzado. This article provides a comprehensive list of the manuscripts of the chronicle, which are then classified into families and subfamilies. An analysis of the sources found in the two main versions of the chronicle (Estoria amplia and Estoria breve) gives rise to a series of hypothesis concerning their relationship and the date of composition of their common archetype. Finally, the article discusses the methodological challenges that this particular historiographical compilation poses to textual scholars, and their wider implications for the study of the late medieval "general chronicles of Spain".

KEYwORDS: Alfonso X, chronicle, De rebus Hispaniae, general chronicles of Spain, Estoria de España, historiography, Estoria del fecho de los godos, Estoria amplia del fecho de los godos, Estoria breve del fecho de los godos, fifteenth century, Jiménez de Rada, textual scholarship, Toledano romanzado. 